\title{
同步辐射及其在无机材料中的应用进展
}

\author{
刘云鹏 ${ }^{1}$ ，盛伟繁 ${ }^{1,2}$ ，吴忠华 ${ }^{1,2}$
}

(1. 中国科学院高能物理研究所, 北京同步辐射实验室, 北京 $100049 ; 2$. 中国科学院大学 核科学与技术学院, 北 京 100049)

摘 要: 同步辐射是环形加速器中做循环运动的高速电子在经过弯转磁铁时, 沿电子轨道切线方向发射的电磁辐 射。作为一类平台型科技基础设施，同步辐射光源对无机材料的研究和发展起到了重要支撑作用。同步辐射实验技 术已经成为现代科学技术不可或缺的研究手段, 无机材料研究是同步辐射技术的主要应用领域之一。相对于用于材 料研究的常规光源来说，同步辐射技术研究无机材料有以下优势：1)获取的数据质量更高; 2)空间分辨和时间分辨 的能力更强; 3)原位和材料服役环境更易模拟; 4)多尺度、多方面、多种类的结构信息同步获取; 5)探测新的结构特 性更有可能。同步辐射实验技术有助于解决无机材料领域中的一些关键科学问题, 从而极大地推动了无机材料的研 究进展。本文首先简要介绍了同步辐射光源的现状，以及国内现有三个同步辐射装置：北京同步辐射装置(Beijing Synchrotron Radiation Facility, BSRF)、上海同步辐射装置(Shanghai Synchrotron Radiation Facility, SSRF)和国家同步 辐射实验室(National Synchrotron Radiation Laboratory, NSRL)。然后, 从 X 射线衍射、散射、谱学、成像等四个方 面, 列举了同步辐射技术在无机材料研究中的应用实例。最后, 对同步辐射光源和结构表征技术及其在无机材料中 的应用进行了总结与展望。

关 键 词: 无机材料; 同步辐射; 光束线; 结构表征; 综述

中图分类号: TQ174 文献标志码: A

\section{Synchrotron Radiation and Its Applications Progress in Inorganic Materials}

\author{
LIU Yunpeng ${ }^{1}$, SHENG Weifan ${ }^{1,2}$, WU Zhonghua ${ }^{1,2}$
}

(1. Beijing Synchrotron Radiation Facility, Institute of High Energy Physics, Chinese Academy of Sciences, Beijing 100049, China; 2. School of Nuclear Science and Technology, University of Chinese Academy of Sciences, Beijing 100049, China)

\begin{abstract}
Synchrotron radiation (SR) is the electromagnetic radiation emitted along the tangent direction of the electron orbit by high-speed electrons moving in a circular accelerator when passing through a bending magnet. SR source, as a platform-type scientific and technological infrastructure, plays an important supporting role in the research and development of inorganic materials. SR techniques become an indispensable research tool of modern science and technology, and inorganic materials are one of the main application fields of SR techniques. Compared with conventional light source used for research, SR techniques in the study of inorganic materials have following obvious advantages: 1) higher obtained data qualities; 2) better spatial and temporal resolutions; 3) easier simulated in-situ and
\end{abstract}

收稿日期：2020-12-08; 收到修改稿日期：2021-01-29; 网络出版日期：2021-04-05

基金项目: 国家重点研发计划(2017YFA0403000); 国家自然科学基金(U1232203)

National Key R\&D Program of China (2017YFA0403000); National Natural Science Foundation of China (U1232203)

作者简介：刘云鹏(1990-), 男, 博士. E-mail: liuyunpeng@ihep.ac.cn

LIU Yunpeng (1990-), male, PhD. E-mail: liuyunpeng@ihep.ac.cn

通信作者: 吴忠华, 研究员. E-mail: wuzh@ihep.ac.cn WU Zhonghua, professor. E-mail: wuzh@ihep.ac.cn 
material service environment; 4) synchronously acquiring multi-scale, multi-faceted and multi-type structural information; 5) new means more likely to detect new structural characteristics. SR techniques help solve some key scientific problems in the field of inorganic materials, and greatly promote their research. Firstly, this paper briefly introduces the current status of SR sources and the three existing domestic SR facilities: Beijing Synchrotron Radiation Facility (BSRF), Shanghai Synchrotron Radiation Facility (SSRF), and National Synchrotron Radiation Laboratory (NSRL). Secondly, some application examples related to inorganic materials research are given from the four aspects of X-ray diffraction, scattering, spectroscopy and imaging. Finally, summary and prospect are given to the SR source, the structural characterization techniques, and their application in inorganic materials.

Key words: inorganic materials; synchrotron radiation; beamline; structure characterization; review

无机非金属材料 ${ }^{[1]}$ (简称无机材料)可简单分为 传统型和新型两类。传统无机材料是指以二氧化硅 及其硅酸盐化合物为主要成分的材料, 如陶瓷、玻 璃、水泥、耐火材料等。传统的无机材料制造业一 直是国民经济的重要支柱产业之一。自 20 世纪 40 年代以来, 由碳、氮、氧、嗍、硫、硅等各种非金 属化合物高纯原料经特殊先进工艺制成的新型无机 材料, 在信息、能源、航空航天、生命科学等现代 科学领域中发挥着极其重要的作用。形成无机材料 的结合键一般为离子键或共价键, 所形成的无机材 料通常具有硬度大、强度高、抗化学腐蚀性强、绝 缘和隔热性良好等特性。随着代表材料制备和应用 的纳米科技 ${ }^{[2]}$ 的快速发展, 以及国民经济日益强劲的 需求, 无机材料的研发方兴未艾。目前, 无机材料研究 的热点和重要方向主要表现在材料的可控制备 ${ }^{[3-4]}$ 、材 料形成 ${ }^{[5-7]}$ 及催化机理研究 ${ }^{[8]}$ 、新相材料的发现 ${ }^{[9]}$ 和 材料特性及应用研究 ${ }^{[10-11]}$ 等方面, 而这些研究方面 的进展都与材料结构关联。事实上, 材料结构与性 能间的关系一直是材料科学的重点研究内容 ${ }^{[12-13]}$ 。 不论是对材料物性机理的研究, 还是对材料性能的 优化提升, 都需要对材料结构有清楚的认识。材料 科学发展的终极目标是准确描述材料的基本规律, 按需设计和制造功能材料, 精准预测材料的服役 寿命和性能的演化, 为此需要对材料的多级结 构、缺陷、相成分与元素含量等诸多因素有更细 致、完备的认识。对于无机材料来说, 包括从电 子结构、原子局域结构、长程有序结构、微纳米 几何结构, 到点缺陷、线缺陷、面缺陷, 再到外场 (热、力、电、磁等) 诱导的结构演化规律都是材料 性能优化、处理工艺改进、新材料探索与设计的 重要依据。材料的结构决定其性能, 对于无机材 料中多尺度各级结构的认识离不开先进的材料结 构探测技术。随着无机材料研究的深入, 传统的
材料结构表征技术越来越难以满足无机材料结构 探测的需求。材料结构优异的时(空)间分辨技术、 友好的原位实时表征手段、先进的不同尺度结构 联合监测能力, 使基于先进同步辐射光源的大科 学装置正成为探究材料微观结构的利器, 贯穿于 材料的合成与服役、结构和性能的研究, 极大地 推动了无机材料学科的发展 ${ }^{[14]}$ 。

本文将对同步辐射光源作简要介绍, 并列举一 些代表性的研究实例, 向读者展示同步辐射实验技 术在无机材料研究中的重要支撑作用。

\section{1 同步辐射光源}

同步辐射 ${ }^{[14]}$ 是环形加速器中做循环运动的高 速电子在经过弯转磁铁时, 沿电子轨道切线方向发 射的电磁辐射, 因美国通用电气公司 1947 年首先在 其同步加速器上观测到而得名 ${ }^{[15]}$ 。据不完全统计, 世界上已有近百台各类同步辐射加速器在运行, 是 当今世界数量最多的一类大科学装置。目前世界上 已建成及在建的高、中、低能区同步辐射光源有近 百个, 主要分布在美洲、欧洲及亚洲。同步辐射光 源的高亮度、高准直性、高稳定性、宽波段覆盖范 围、高偏振性和脉冲时间结构等诸多优异性能 ${ }^{[16-19]}$, 使得同步辐射光源逐步取代了常规的 $\mathrm{X}$ 射线源, 发 展成为现代科学研究的集散地。相较于传统实验表 征技术与方法, 依托于同步辐射光源的表征技术与 方法具有很多优势，并演化出能量色散、实时原位、 时间分辨、动态演化等当代科技研究不可或缺的重 要表征手段。同步辐射光源及其实验技术已在物理、 化学、化工、材料、生物、医学、地矿、农林、考 古、微加工技术等诸多学科与领域发挥了重要的作 用 ${ }^{[20-24]}$, 特别是对于无机材料而言, 同步辐射光源 与技术已成为极为重要的研究工具。 


\section{2 国内同步辐射装置}

我国大陆已建成并投入使用的同步辐射光源共有 三个, 分别是北京同步辐射装置(Beijing Synchrotron Radiation Facility, BSRF) ${ }^{[25]}$ 、上海同步辐射装置 (Shanghai Synchrotron Radiation Facility, SSRF) ${ }^{[26]}$ 以 及合肥国家同步辐射实验室(National Synchrotron Radiation Laboratory, NSRL) ${ }^{[27]}$ 。BSRF 是我国建成 的第一个同步辐射装置, 始于北京正负电子对撞机 (Beijing Electron-Positron Collider, BEPC), 属于第 一代同步辐射光源。BEPC 和 BSRF 经过二期改进 后, 同步辐射品质有所提高, 大致相当于一代半的 同步辐射光源。BSRF 由于与 BEPC 共用相同的储 存环, 其运行分兼用和专用两种模式: 在兼用模式 下，储存环以高能物理正负电子对撞实验为主，储 存环电子束流能量运行在 1.6 2.8 GeV 之间, 电子 束发射度约 $390 \mathrm{~nm} \cdot \mathrm{rad}\left(1 \mathrm{rad}=(180 / \pi)^{\circ}\right)$; 在同步辐 射专用模式下，储存环电子束流能量运行在 $2.5 \mathrm{GeV}$, 电子束发射度约 $80 \mathrm{~nm} \cdot \mathrm{rad}$ 。BSRF 提供低中能量段 的同步辐射光束, 以中能量段的硬 $\mathrm{X}$ 射线为主。 NSRL 是我国建成的第一个第二代同步辐射光源, 其储存环电子束流能量运行在 $0.8 \mathrm{GeV}$, 电子束发 射度小于 $40 \mathrm{~nm} \cdot \mathrm{rad}$ 。NSRL 提供中低能量段的同步 辐射, 侧重于软 $X$ 射线和真空紫外波段光源, 适于 向波长更长的红外、远红外波段扩展。SSRF 是我 国建成的第一个第三代同步辐射光源, 其储存环电 子束流能量运行在 $3.5 \mathrm{GeV}$, 电子束发射度约 $3.9 \mathrm{~nm} \cdot \mathrm{rad}$ 。SSRF 能够提供低中高能量段的广谱同 步辐射, 以插入件(扭摆器、波荡器)引出的中能量段 硬 X 射线为主。此外, 我国台湾新竹市也有两台三 代同步辐射光源: 一台电子束流能量运行在 $1.5 \mathrm{GeV}$, 电子束发射度为 $25.6 \mathrm{~nm} \cdot \mathrm{rad}$ 的低能光源 (Taiwan Light Source, TLS), 更适合于开展软 X 射 线和真空紫外波段的研究; 另一台电子束流能量运 行在 $3 \mathrm{GeV}$, 电子束发射度为 $1.5 \mathrm{~nm} \cdot \mathrm{rad}$ 的中能光 源(Taiwan Photon Source, TPS), 是一个典型的三代 中能同步辐射光源。近年来, 我国同步辐射光源发 展势头强劲, 北京正在建设位于高能区 $(6 \mathrm{GeV})$ 的 基于衍射极限储存环的第四代同步辐射光源，即高 能同步辐射光源 (High Energy Photon Source, HEPS)。上海正在对 SSRF 进行线站工程二期建设。 合肥也正在设计位于中低能区 $(\sim 2 \mathrm{GeV})$ 的基于衍射 极限储存环的第四代同步辐射光源, 即合肥先进光 源(Hefei Advanced Light Facility, HALF)。另外, 还 有一些同步辐射光源正在计划或酝酿之中。

\section{3 同步辐射技术在无机材料中的应用}

材料的物性取决于材料的物质结构, 基于同步 辐射光源的物质结构表征技术在现代科学研究中起 着不可替代的作用。这一方面得益于同步辐射光源 相对于传统的实验室 $\mathrm{X}$ 射线源 $(\mathrm{X}$ 光机)在强度、亮 度、谱宽、准直性、偏振性、时间结构等诸多方面 具有无法比拟的优势，另一方面也得益于同步辐 射光源上集中了各种各样的物质结构表征技术, 使原位、实时、联用等先进实验技术用于物质结构 的表征成为可能。除此之外，同步辐射装置及其结 构表征技术还有共享的特性。几乎世界上所有的同 步辐射光源都是公益性的大科学装置, 面向全国 乃至全球的高等院校、科研院所, 免费使用, 高度 共享, 开放运行。当然, 作为用户要想获得同步辐 射光源上的实验机时, 需要向相应的同步辐射光 源申请实验课题, 而同步辐射光源将组织专家对 用户申请的课题进行评审，择优支持申请通过的 用户，并向其分配实验机时。同步辐射结构表征技 术随着同步辐射光源的进步而不断发展, 各种新 的实验技术也在不断完善，探测物质结构的能力 也在不断提高。同步辐射技术在无机材料方面的应 用非常广泛，为了更好地了解同步辐射实验技术 在无机材料结构表征方面的应用，以下将从衍射、 散射、谱学、成像等典型的应用方面列举若干实例 予以说明。

\section{1 衍射应用示例}

$X$ 射线衍射(X-ray Diffraction, XRD)是研究物 质周期性结构的主要手段。相比于用传统的转靶 X 射线衍射仪做 XRD 而言, 基于同步辐射光源的 $\mathrm{XRD}$ 技术的光源强度要高几个量级, 可在较宽的范 围内任选入射 $\mathrm{X}$ 射线的能量或波长, 通常能够快速 精确地得到高信噪比、高角分辨、高能量分辨的 $X R D$ 数据。特别是在同步辐射 $X$ 射线衍射实验站 上, 原位实时开展无机材料结构的研究已成为常 态。结合 Rietveld 结构精修 ${ }^{[28]}$ 等方法, 同步辐射 X 射线衍射技术用于研究无机材料晶体结构随外加 条件的变化, 对于评估或改善材料的性能都具有 重要的帮助作用。

影响无机材料晶体结构最常见的外部因素主要 有温度和压力。利用 XRD 技术可以获取不同外场 (如温度、压力等)处理下无机材料的非原位相结构 ${ }^{[4-5]}$ 及可能的变化。利用同步辐射极端条件下的 XRD 技术, 可以研究原位高压条件下的材料晶体结构演 化 ${ }^{[29-31]}$, 如果再给材料加上温度, 还可研究材料的 
状态方程 ${ }^{[32]}$ 。Fang 等 ${ }^{[33]}$ 在 BSRF 的 4W2 线站用原 位同步辐射高压衍射技术研究二维卤化物双钻钛矿 $(\mathrm{BA})_{4} \mathrm{AgBiBr}{ }_{8}\left(\mathrm{BA}=\mathrm{CH}_{3}\left(\mathrm{CH}_{2}\right)_{3} \mathrm{NH}_{3}{ }^{+}\right)$的结构与光学 性能之间的关系, 发现压力为 $2.5 \mathrm{GPa}$ 时出现了压 力诱导的光发射, 压力增至 $8.2 \mathrm{GPa}$ 时光发射强度 进一步显著增强, 压力高达 $25 \mathrm{GPa}$ 时能隙从 $2.61 \mathrm{eV}$ 变窄到 $2.19 \mathrm{eV}$, 且发射光由浅黄色变成深黄色。研 究结果表明这些光学性能的变化与压力诱导的相变 有关, 在压力诱导下 $\left[\mathrm{AgBr}_{6}\right]^{5-}$ 和 $\left[\mathrm{BiBr}_{6}\right]^{3-}$ 八面体之 间发生倾转, Bi-Br1-Ag 键发生弯曲, 如图 1 所示。这 一研究对于改善二维双钙钛矿材料的光学性能具有 重要的指导意义。

在充放电过程中, 电池正负极材料结构的变化 或中间产物物相与成分的确定对于实现大容量阳极 材料、高能量密度电池具有指导意义, 常规 XRD 技 术 $^{[34]}$ 在这样一些物质结构的测量中也能发挥重要 作用。时间分辨的 X 射线衍射技术用于监测无机材 料晶体结构的动态演变过程更是不可或缺。中国科 学院大学的刘向峰课题组 ${ }^{[35-36]}$ 利用北京同步辐射装 置 4B9A 束线 $X$ 射线衍射站所装配的一维弧形 Mythen 探测器 ${ }^{\left[{ }^{[37-38]}\right.}$ 对 $\mathrm{Li}^{-} \mathrm{O}_{2}$ (或 $\mathrm{Na}^{-} \mathrm{O}_{2}$ ) 电池原位放
电过程中 $\mathrm{Co}_{3} \mathrm{O}_{4}$ 纳米片 (或 $\mathrm{CoO} / \mathrm{CoP}$ 异质结构纳米 片) 电催化剂的结构演变开展研究, 揭示了放电过 程中电催化过程以及反应产物的物相演变。这些时 间分辨的动态过程研究对于可充电电池的高性能无 机催化剂材料设计具有重要意义。

$\beta-\mathrm{CsPbI}_{3}$ 具有适用太阳能电池的带隙, 但是要 沉积制备出结构稳定的高性能 $\beta-\mathrm{CsPb}_{3}$ 薄膜仍然是 个挑战。以往只能在 $230{ }^{\circ} \mathrm{C}$ 以上观测到 $\beta-\mathrm{CsPbI}_{3}$ 物 相, 而 Wang 等 ${ }^{[11]}$ 制备出了光谱响应范围宽、物相 稳定、结晶度高的 $\beta-\mathrm{CsPbI}_{3}$ 薄膜。用 XRD 技术和 同步辐射掠入射广角散射(Grazing Incidence Wide Angle Scattering of Synchrotron Radiation, GIWAXS) 技术不仅确定了其物相(如图 2 所示), 并且证实该 薄膜由高度取向的 $\beta-\mathrm{CsPbI}_{3}$ 晶粒构成, 结合质谱分 析证实薄膜的成分全是无机的。为了延长载流子寿 命和改善 $\beta-\mathrm{CsPbI}_{3}$ 吸收层与载流子选择接触体间的 能级匹配, 用碘化胆碱表面处理薄膜减缓了钙钛矿 层中裂纹和针孔的影响。这种处理后的材料制成的 钻钛矿太阳能电池在 $(45 \pm 5){ }^{\circ} \mathrm{C}$ 的环境条件下, 其光 电转化效率(Photon-to-electron Conversion Efficiency, PCE)超过了 $18 \%$ 。
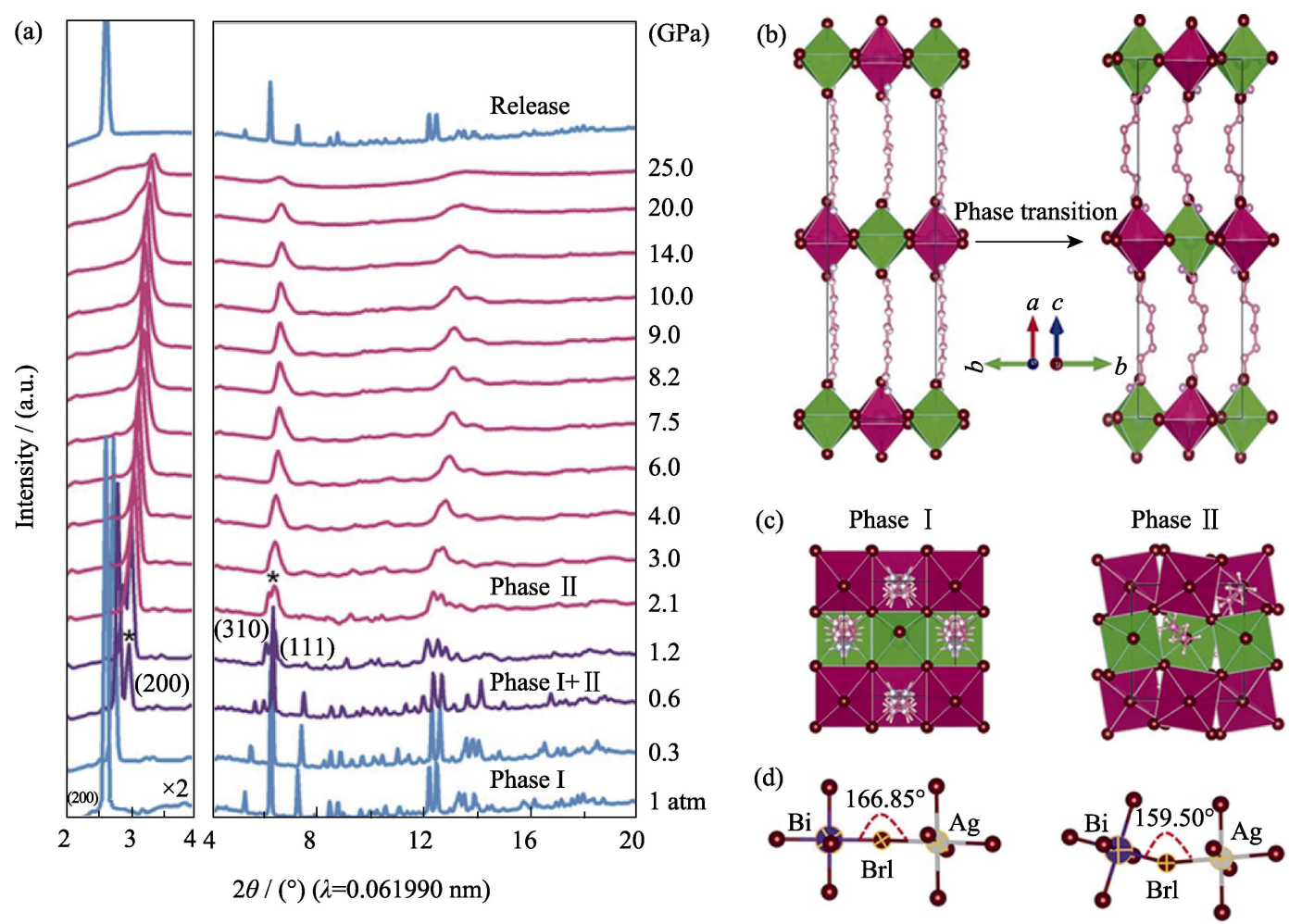

图 $1 \quad(\mathrm{BA})_{4} \mathrm{AgBiBr}_{8}$ 的原位高压衍射图谱及其晶体结构变化示意图 ${ }^{[33]}$

Fig. 1 In situ high pressure $\mathrm{XRD}$ patterns of $(\mathrm{BA})_{4} \mathrm{AgBiBr}_{8}$ and schematic illustration of crystal structure changes ${ }^{[33]}$ (a) Representative XRD patterns of $(\mathrm{BA})_{4} \mathrm{AgBiBr}_{8}$ at selected pressures up to $25.0 \mathrm{GPa}$. The magnification factor of the peak intensities in the left panel is two times that of the right panel; (b) Crystal structure of (BA) ${ }_{4} \mathrm{AgBiBr}_{8}$ perpendicular to the $c$ axis (perpendicular to $a$ axis, phase II). Pink and green octahedra represent $\left[\mathrm{AgBr}_{6}\right]^{5-}$ and $\left[\mathrm{BiBr}_{6}\right]^{3-}$ octahedra, respectively; (c) Crystal structures of $(\mathrm{BA})_{4} \mathrm{AgBiBr}_{8}$ along $(100)((001), \mathrm{Phase} \mathrm{II})$ with increasing pressure. $\mathrm{Ag}-\mathrm{Br}$ octahedra are shown in pink. $\mathrm{Bi}-\mathrm{Br}$ octahedra are shown in green; (d) Illustrations of $\mathrm{Bi}-\mathrm{Br} 1-\mathrm{Ag}$ bond angle within the inorganic layers framework before and after the phase transition $\left(1 \mathrm{~atm}=1.01 \times 10^{5} \mathrm{~Pa}\right)$ 

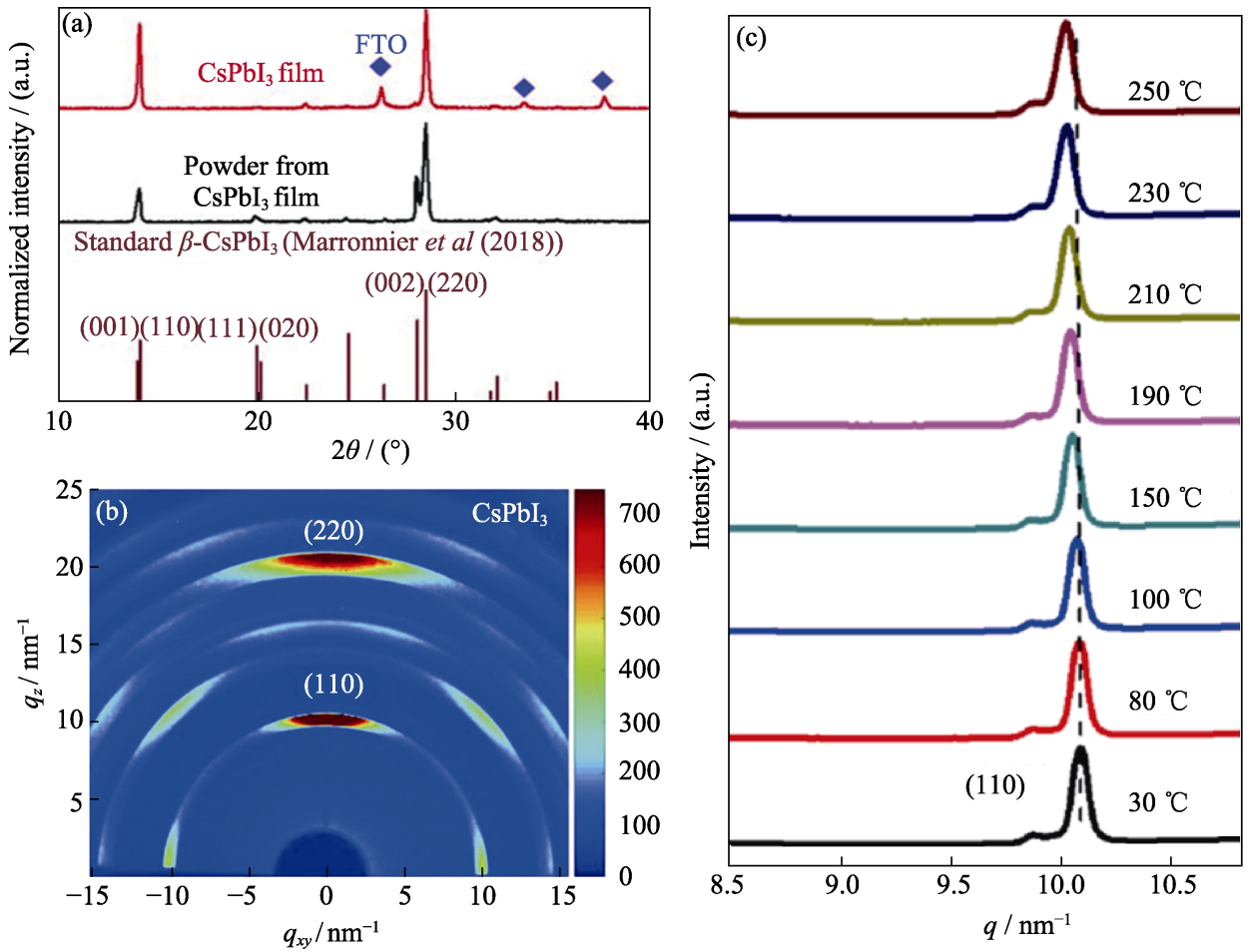

图 $2 \beta-\mathrm{CsPbI}_{3}$ 的同步辐射 $\mathrm{X}$ 射线衍射的相关谱图 ${ }^{[11]}$

Fig. 2 Relevant SR-XRD patterns of $\beta$ - $\mathrm{CsPbI}_{3}{ }^{[11]}$

(a) XRD patterns acquired from a $\mathrm{CsPbI}_{3}$ thin film and powders scratched from its films. Brown lines indicate the standard $\beta$-CsPbI ${ }_{3} \mathrm{XRD} \mathrm{pattern}$ calculated for $\mathrm{Cu} \mathrm{K} \alpha_{1}$ radiation for the tetragonal perovskite structure at $518 \mathrm{~K}$; (b) GIWAXS data from $\beta$ - $\mathrm{CsPbI}_{3}$ films; (c) Azimuthally integrated intensity profiles for the $\beta-\mathrm{CsPbI}_{3}$ perovskite films annealed at different temperatures $\left(30,80,100,150,190,210,230,250{ }^{\circ} \mathrm{C}\right)$

热电技术能够收集废热并将其直接转化为电能, 北京航空航天大学赵立东团队 ${ }^{[39]}$ 研究了 $\mathrm{SnSe}$ 的热 电性能。已往的研究表明层状材料的热导率在平面 外方向最低(2D 声子输运), 而用 $\mathrm{Br}$ 掺杂制备的 $\mathrm{n}$ 型 $\mathrm{SnSe}$ 晶体在 $773 \mathrm{~K}$ 下的最大热电优值 $(Z T)$ 达到 $(2.8 \pm 0.5)$ 。这是因为 $\mathrm{Sn}$ 和 $\mathrm{Se}$ 的 $\mathrm{p}$ 电子在导带最小 值附近离域 $(\mathrm{CBM})$ 有助于更多的轨道交叠出平面 $(b c)$ 的方向 ( $a$ 轴方向), 使 $\mathrm{n}$ 型 $\mathrm{SnSe}$ 晶体具有交叠的 层间电荷密度(3D 电荷传输)。他们利用原位高温同 步辐射 XRD 技术观察到 $\mathrm{n}$ 型 $\mathrm{SnSe}$ 晶体经历了一个 连续地从 Pnma 到 $\mathrm{Cmcm}$ 的相变, 该相变开始于临 界温度 $(800 \mathrm{~K})$ 以下的 $600 \mathrm{~K}$ 左右, 如图 3 所示。XRD 结果揭示连续的相变提高了 $\mathrm{n}$ 型 $\mathrm{SnSe}$ 晶体的对称性, 分散了两个会聚的导带, 提高了载流子迁移率, 同 时保持了较大的泽贝克系数。这一研究为探索新型 高效热电材料提供了新思路, 并为二维层状材料提 供了一种增强平面外电输运性能但不降低热性能的 新策略。

$X$ 射线衍射技术在解析无机材料未知晶相结构 方面总是扮演着举足轻重的角色，特别是同步辐射 光源的应用, 基于同步辐射装置的 XRD 技术已成 为包括无机材料在内的各种物质结晶结构测定的首 选方法。碳酸钙 $\left(\mathrm{CaCO}_{3}\right)$ 是一种在建筑行业常用的无
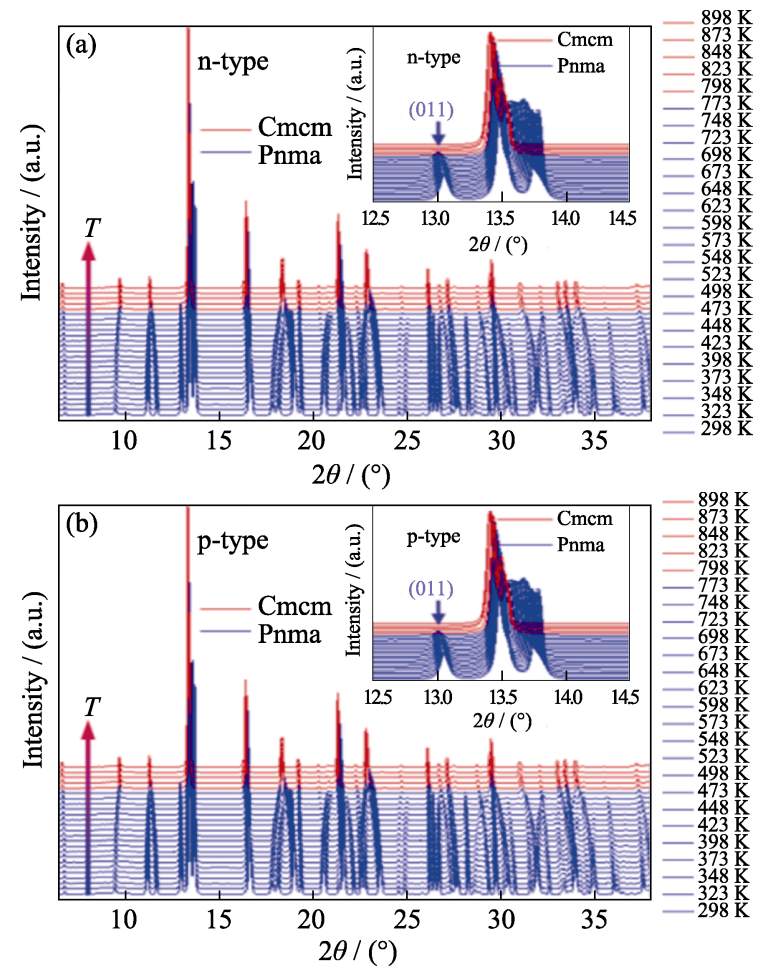

图 $3 \mathrm{n}$ 型 $(\mathrm{a})$ 和 $\mathrm{p}$ 型 (b)SnSe 的原位高温 $\mathrm{X}$ 射线衍射图谱 ${ }^{[39]}$ Fig. 3 In-situ high temperature XRD patterns of n-type (a) and p-type (b) $\mathrm{SnSe}^{[39]}$

Colorful figures are available on website

机材料, 也是各种海洋生物骨架和外壳的主要成 分。 $\mathrm{CaCO}_{3}$ 不仅在全球碳循环中扮演着重要的角色, 
而且在沉积岩的形成中发挥重要的作用。来自德国 普朗克胶体和界面研究所的 Zou 及其合作者 ${ }^{[9]}$ 在研 究非晶碳酸钙结晶过程中 $\mathrm{Mg}^{2+}$ 扮演的角色时, 意外 地发现了一个未知物相。已知碳酸钙在环境条件下 只存在方解石、霰石和球霰石三种纯 $\mathrm{CaCO}_{3}$ 晶型, 以 及一水方解石 $\left(\mathrm{CaCO}_{3} \cdot \mathrm{H}_{2} \mathrm{O}\right)$ 和六水方解石 $\left(\mathrm{CaCO}_{3} \cdot 6 \mathrm{H}_{2} \mathrm{O}\right)$ 两种水合结晶相。Zou 等利用同步辐射 XRD 技术解 析出该未知物相具有单斜结构, 为半水碳酸钻晶相 $\left(\mathrm{CaCO}_{3} \cdot 1 / 2 \mathrm{H}_{2} \mathrm{O}\right)$, 如图 4 所示。这一发现对生物矿 化、地质学和工业过程有重要的意义。

\section{2 散射应用示例}

散射是 $\mathrm{X}$ 射线与物质相互作用的主要形式之 一。从起源上来说, $\mathrm{X}$ 射线与物质相互作用过程中不 引起 $X$ 射线能量改变的过程大都可归为 $X$ 射线受物 质中电子散射的作用过程，比如 $\mathrm{X}$ 射线衍射现象就 发生在被物质(此时为晶体)不同部位(此时为晶面) 散射的 $X$ 射线在某个散射方向上光程差为波长的整 数倍或相位差为 $2 \pi$ 的整数倍。从这种意义上来说, $\mathrm{X}$ 射线散射是普遍的, 对任何形态的物质都可能发生
$X$ 射线散射; 而 $X$ 射线衍射仅是一种特殊情况下的 散射结果, 仅具有周期结构的物质或晶体才可能出 现 $X$ 射线衍射。小角 $X$ 射线散射 (SAXS) 是最具代 表性的 X 射线散射应用之一。在 20 世纪 30 年代初, Krishnamurti $^{[40]}$ 和 Warren ${ }^{[41]}$ 首先发现了 SAXS 现象。 此后, Guinier 等 ${ }^{[42]}$ 建立并发展了 SAXS 理论。随着 同步辐射光源的应用, 同步辐射小角 $X$ 射线散射 (SR-SAXS) 技术得到了广泛的应用。SAXS 技术通 常用于研究物质内部等同电子密度区域(或颗粒)大 小约 1 100 nm 的几何结构; 所适用的样品状态可 以是固体、粉末、胶体和液体; 样品中的颗粒可以 是有序排列, 亦可以是完全无序分布。SAXS 技术 已被广泛用于探测无机纳米材料、介孔材料、薄膜 等多散体系的纳米颗粒粒径或纳米孔径大小分布, 也被用于测定聚合物、纳米周期结构, 还被用于分 析生物蛋白等单分散体系的散射体(颗粒、蛋白分子) 形状。可以说, 同步辐射 SAXS 实验站是同步辐射 装置用户最多的实验站之一。

利用原位动态的 SAXS 技术 ${ }^{[43-46]}$ 可研究无机材
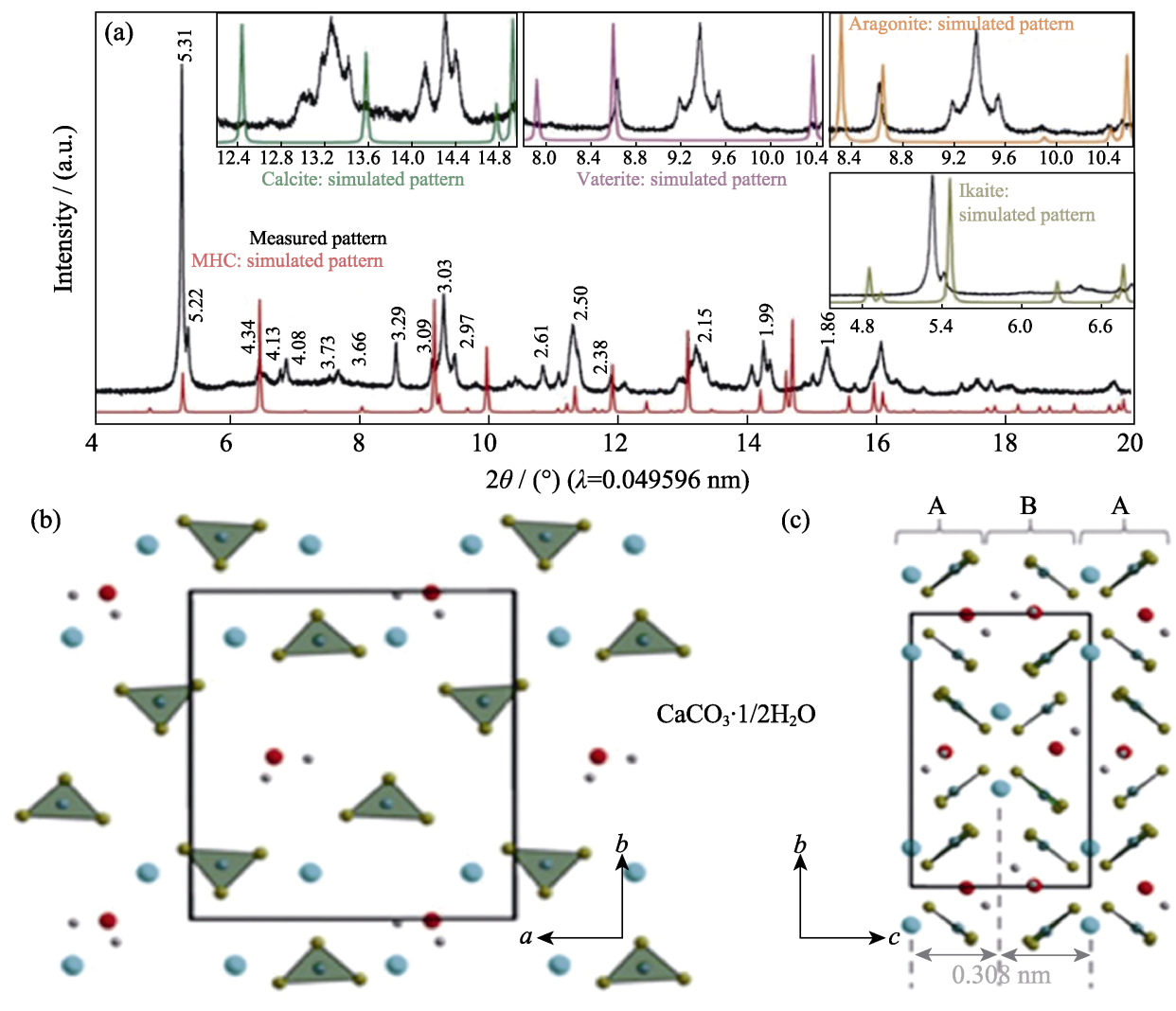

图 $4 \mathrm{CaCO}_{3} \cdot 1 / 2 \mathrm{H}_{2} \mathrm{O}$ 的高分辨同步辐射 $\mathrm{X}$ 射线衍射图谱及其晶体结构模型 ${ }^{[9]}$

Fig. 4 High-resolution SR-XRD pattern of $\mathrm{CaCO}_{3} \cdot 1 / 2 \mathrm{H}_{2} \mathrm{O}$ and its crystal structure model ${ }^{[9]}$ (a) Synchrotron HRXRPD pattern of $\mathrm{CaCO}_{3} \cdot 1 / 2 \mathrm{H}_{2} \mathrm{O}$ using a wavelength of $0.049596 \mathrm{~nm}$; (b) The layers of $\mathrm{CaCO}_{3} \cdot 1 / 2 \mathrm{H}_{2} \mathrm{O}$ consist of $\mathrm{Ca}\left(\mu 2-\mathrm{CO}_{3}\right) 2 / 5\left(\mu 1-\mathrm{CO}_{3}\right) 3 / 5\left(\mathrm{H}_{2} \mathrm{O}\right) 1 / 2$ double zigzag chains running along the $a$ axis, here, differently oriented (up and down) carbonate groups are indicated by indices $\mu 2$ and $\mu 1$; (c) Stacking in the $c$ direction occurs in a conventional ABAB fashion with an interlayer spacing (the distance between Ca planes) equal to $0.308 \mathrm{~nm}$. The insets in (a) show comparisons of the measured HRXRPD pattern of $\mathrm{CaCO}_{3} \cdot 1 / 2 \mathrm{H}_{2} \mathrm{O}$ with simulated patterns of other calcium carbonate phases. Different types of atoms are colored as follows: $\mathrm{Ca}$, blue; carbonate group, green; oxygen from water, red; and hydrogen, gray 
料合成过程中的颗粒形核、生长、演化和结构转变 过程。YAN 等 ${ }^{[43-45]}$ 利用原位动态 SAXS 技术对 Ag 纳米材料的形核生长过程进行了研究。在研究 $\mathrm{Ag}$ 纳米颗粒的形核生长过程时 ${ }^{[43]}$, 分别对四种初始 $\mathrm{Ag}^{+}$浓度为 $2 、 4 、 6$ 和 $8 \mathrm{mmol} / \mathrm{L}$ 的反应溶液进行了 时间分辨的 SAXS 研究, 从实验上确定了四种 $\mathrm{Ag}^{+}$ 初始浓度下 $\mathrm{Ag}$ 纳米颗粒的形核半径, 以及 $\mathrm{Ag}$ 纳米 颗粒大小随时间的变化。依据实验结果，提出了 $\mathrm{Ag}$ 纳米颗粒生长的扩散团聚 (Diffusion-coalescence Model, DC Model)模型如图 5 所示。该模型的主要 思路是先形成的银核或颗粒在布朗运动驱使下在溶 液中扩散，使银颗粒间有一定概率互相碰撞而结合 长大。基于这一思路导出了 $\mathrm{Ag}$ 颗粒尺寸 $R$ 随反应 时间 $t$ 的变化如下:

$$
R=r_{\mathrm{c}}+\frac{r_{0}}{b}\left(C_{\mathrm{Ag}} N_{\mathrm{A}}\right)^{1 / 3} \ln \left[1+\frac{8 b r_{0}}{3 r_{\mathrm{c}}} Q\left(r_{\mathrm{c}}\right)\left(C_{\mathrm{Ag}} N_{\mathrm{A}}\right)^{1 / 3}\left(t-t_{\mathrm{c}}\right)\right]
$$

式中, $C_{\mathrm{Ag}}$ 为溶液中 $\mathrm{Ag}^{+}$的初始浓度, $N_{\mathrm{A}}$ 为阿伏伽德 罗常数, $r_{0}$ 为反应前 $\mathrm{Ag}$ 原子或 $\mathrm{Ag}^{+}$离子的平均半径, $r_{\mathrm{c}}$ 为临界形核半径, $b$ 为与溶液相关的衰减因子, $Q\left(r_{\mathrm{c}}\right)$ 为耦合相关函数, 与颗粒的扩散系数 $D$, 颗粒 生长模式因子 $z(z>1)$, 以及两个或多个核(小颗粒) 结合成一个大颗粒的概率函数 $P\left(r_{\mathrm{c}}\right)$ 有关。在四种 不同初始浓度下，该模型计算的 $\mathrm{Ag}$ 颗粒尺寸与 SAXS 实验测得的 Ag 颗粒尺寸互相吻合, 并且发

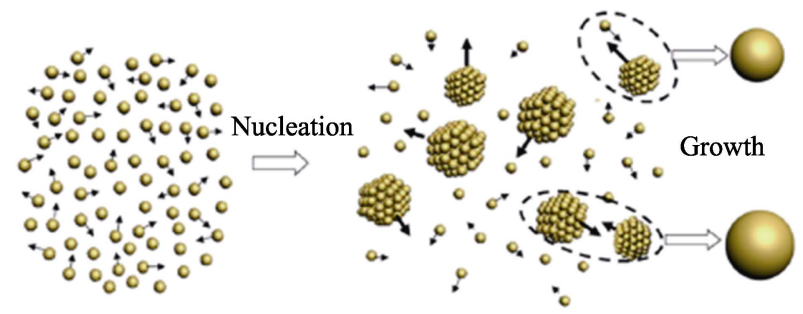

图 $5 \mathrm{Ag}$ 纳米颗粒的扩散聚集生长机制示意图 ${ }^{[43]}$

Fig. 5 Sketch map of the diffusion-coalescence mechanism of Ag nanoparticles ${ }^{[43]}$
现颗粒最终的尺寸与初始的反应浓度并不是线性相 关的。 $8 \mathrm{mmol} / \mathrm{L}$ 的溶液虽然初始浓度相对较高, 但 形核半径与碰撞结合率都比较低, 最终导致该溶液 中的颗粒尺寸较小。

YAN 等 ${ }^{[45]}$ 还利用同步辐射原位 XRD 和原位 SAXS 技术研究了 PVP 包裹的 Ag 纳米粒子的加热 过程。用谢乐公式分析 XRD 图谱衍射峰半峰全宽 的变化可以获得样品中晶粒尺寸的变化，结合 SAXS 数据分析还可以给出样品中颗粒大小的变 化。结合 XRD 和 SAXS 结果, 发现 PVP 包裹的 Ag 纳米颗粒在 $400{ }^{\circ} \mathrm{C}$ 以下即 PVP 完全分解之前, $\mathrm{Ag}$ 纳米粒子长大表现为缓慢的奥斯瓦尔德熟化过程, [111]方向为易生长方向，每个颗粒大致包含一个晶 粒。在 400 500 ${ }^{\circ} \mathrm{C}$ 之间, PVP 已完全分解, $\mathrm{Ag}$ 纳米 粒子的长大表现为快速的奥斯瓦尔德熟化过程, [200]方向为易生长方向，每个颗粒大致仍只包含一 个晶粒。在 500 600 ${ }^{\circ} \mathrm{C}$ 之间, $\mathrm{Ag}$ 纳米粒子的长大表 现为随机的团聚过程，每个颗粒包含有若干取向不 同的晶粒。然而当温度进一步升至 $600 \sim 700{ }^{\circ} \mathrm{C}$ 时， $\mathrm{Ag}$ 纳米粒子长大表现为定向融合生长过程, 每两 个晶粒沿[200]方向融合聚集成一个花生状的大晶 粒。在 $700{ }^{\circ} \mathrm{C}$ 以上，一个颗粒又仅包含一个已融合 好的晶粒。这一研究揭示了 $\mathrm{Ag}$ 纳米晶粒在加热过 程中通过旋动与平动达到定向融合聚集长大的机制, 如图 6 所示。

为了解析 $\mathrm{Ag}$ 花生状纳米颗粒的结构, 该研究 还首次导出了其回转半径 $(l)$ 的表达式如下:

$$
r_{\mathrm{g}}(R, l)=\left(\frac{12 R^{5}+30 R^{4} l+20 R^{3} l^{2}+2 l^{5}}{20 R^{3}+30 R^{2} l-10 l^{3}}\right)^{1 / 2}
$$

纳米颗粒的聚集生长是一种比较常见的生长模 式。谭园园等 ${ }^{[46]}$ 在 $\mathrm{BSRF}$ 的 $1 \mathrm{~W} 2 \mathrm{~A}$ 线站通过原位 SAXS 表征手段研究了脉冲放电制备 NiP 纳米颗粒

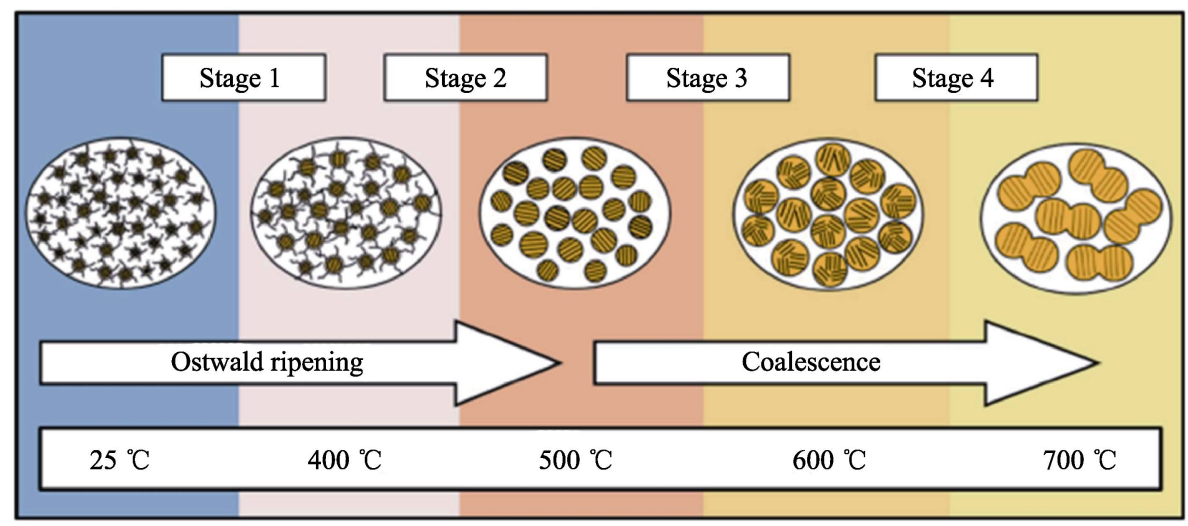

图 $6 \mathrm{PVP}$ 包裹的 $\mathrm{Ag}$ 纳米颗粒的定向聚集生长机制示意图 ${ }^{[45]}$

Fig. 6 Sketch of the growth process of PVP-coated silver nanoparticles ${ }^{[45]}$ 
的过程。SAXS 结果表明在反应最初 $60 \mathrm{~s}$ 时间内, 体 系的颗粒尺寸呈现单峰分布, 且峰的宽度随时间的 变化逐渐增大, $\mathrm{NiP}$ 纳米粒子的长大主要通过 $\mathrm{Ni}$ 和 $\mathrm{P}$ 原子的扩散方式进行。当反应进行到 90 180 s 时, 颗粒尺寸呈现双峰分布, 且尺寸较小的那个峰位符 合反应初期的单峰峰位变化趋势, 尺寸较大的峰位 是尺寸较小的峰位宽度的两倍以上。这种颗粒尺寸 的双峰分布揭示体系中出现了新的多颗粒集聚生长 方式。当反应时间继续延长到 $200 \mathrm{~s}$ 以上时, 颗粒尺 寸分布又呈单峰分布, 说明通过前一阶段的聚集生 长后, 溶液中颗粒浓度降低, 多颗粒集聚生长的条 件较难满足, $\mathrm{Ni}$ 和 $\mathrm{P}$ 原子扩散又成为主要的生长方 式。这一研究结合 XAFS 分析给出了 $\mathrm{NiP}$ 纳米颗粒 随反应时间的结构演变和尺寸分布变化, 并提出了 $\mathrm{NiP}$ 纳米颗粒从结晶 $\mathrm{Ni}$ 形核 $\rightarrow \mathrm{Ni}-\mathrm{P}$ 形核 $\rightarrow$ 结晶 $\mathrm{Ni}$ 向非晶 Ni-P 转变 $\rightarrow$ 非晶 Ni-P 颗粒生长的四步形成 机制, 如图 7 所示。这一研究对于无机化合物纳米 粒子相结构、粒度和组成的有效控制具有一定的指
导意义。

SAXS 技术常用于表征无机纳米材料纳米尺寸 的颗粒大小与分布, 在生物蛋白等单分散体系的应 用方面主要是颗粒形状的获取。SAXS 技术的另一 个应用是探测各向异性纳米颗粒的空间取向分布。 王佳怡等 ${ }^{[47}$ 利用原位 SAXS 技术研究了硅油悬浮液 中 $\mathrm{Na}_{2} \mathrm{Ti}_{3} \mathrm{O}_{7}$ 无机纳米纤维在外加电场作用下的取向 变化, 获取了不同浓度下纳米纤维取向度随外加电 场强度的变化, 如图 8 所示。悬浮液中纳米纤维取 向度随电场和浓度的变化可以用一个经验公式来描 述。将无机纳米颗粒均匀分散在介电液体中形成的 悬浮液有可能成为性能优异的电流变液材料并具有 潜在的应用前景, 因而研究分散的无机纳米颗粒在 外部电场影响下的响应行为, 比如分散相颗粒的团 聚、结构相变、取向变化等, 有望为揭示电流变液 微观结构响应机制和力学性能的理论预测提供新的 线索, 为电流变液在智能控制领域的发展奠定理论 基础。
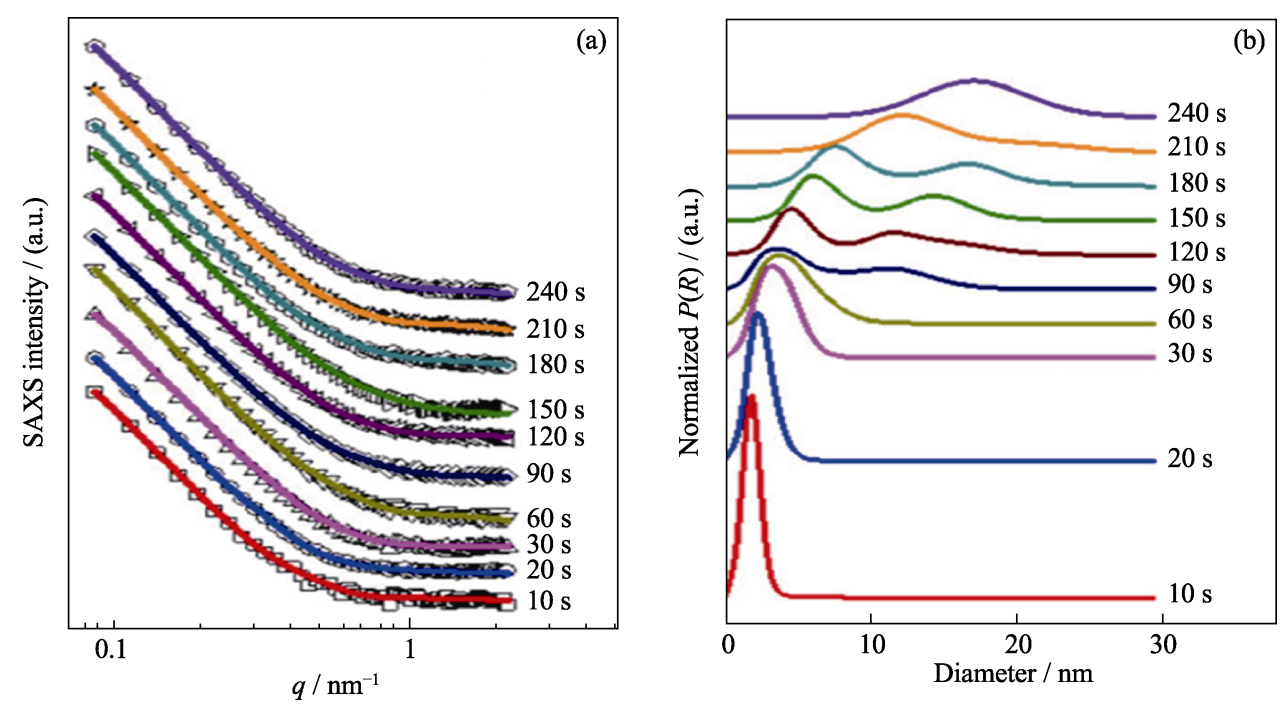

(c)

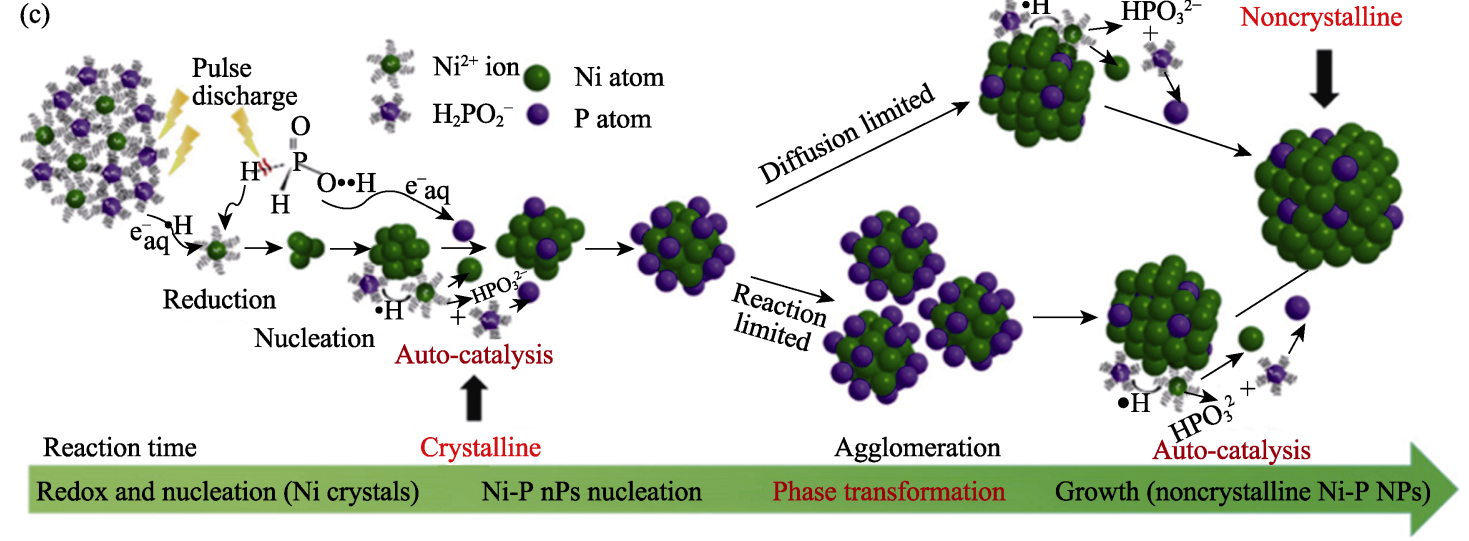

图 7 时间分辨 SAXS 实验结果及 NiP 纳米颗粒形成机理示意图 ${ }^{[46]}$

Fig. 7 Time-resolved SAXS results and schematic illustration of the formation mechanism for $\mathrm{NiP}^{[46]}$ (a) Comparison between the experimental SAXS intensities (symbols) and the simulated ones (solid lines); (b) Normalized particle-volume distribution $P(R)$ extracted from the SAXS data; (c) Schematic map of the formation mechanism of noncrystalline NiP nanoparticles 
(A)

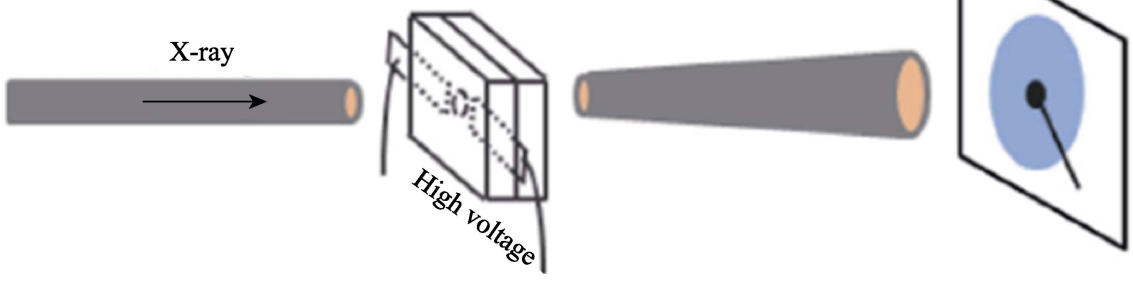

(B)
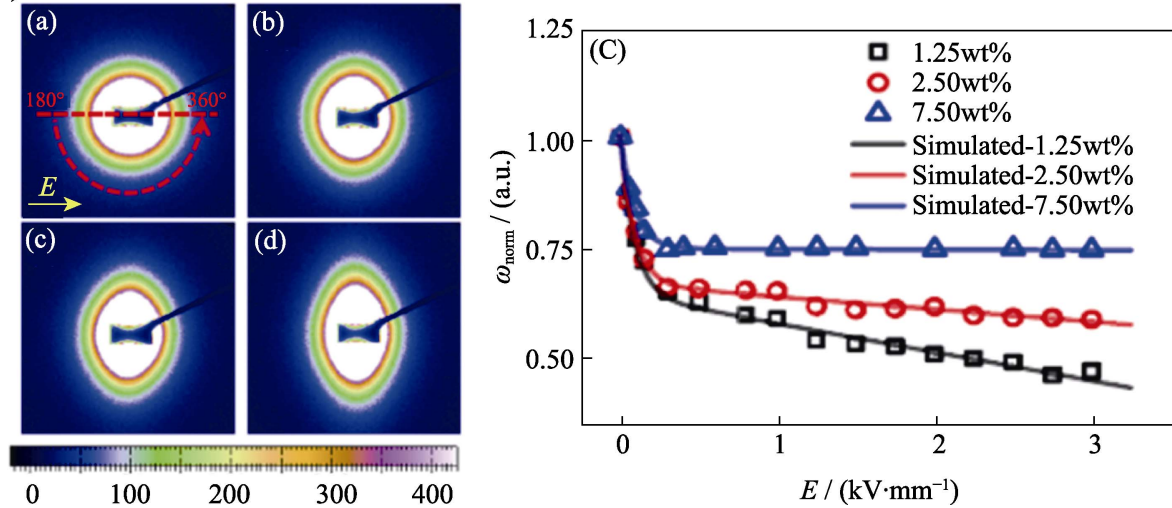

图 8 原位 SAXS 技术研究悬浮的外加电场诱导的 $\mathrm{Na}_{2} \mathrm{Ti}_{3} \mathrm{O}_{7}$ 纳米纤维的取向行为 ${ }^{[47]}$

Fig. 8 In-situ SAXS technique applied on the study for the orientation of suspended $\mathrm{Na}_{2} \mathrm{Ti}_{3} \mathrm{O}_{7}$ nanofiber induced by applied electric field ${ }^{[47]}$ (A) Sketch map of in-situ SAXS measurements; (B) SAXS patterns of the silicone oil suspension with $1.25 \mathrm{wt} \%$ of $\mathrm{Na}_{2} \mathrm{Ti}_{3} \mathrm{O}_{7}$ nanofibers under the DC electric-field strength: $0 \mathrm{kV} / \mathrm{mm}$ (a), $0.5 \mathrm{kV} / \mathrm{mm}$ (b), $1.5 \mathrm{kV} / \mathrm{mm}$ (c), and $2.5 \mathrm{kV} / \mathrm{mm}$ (d); (C) Electric-field strength dependence of normalized orientation distribution width, the solid lines represent the simulation

SAXS 技术用于纳米尺度周期性结构的研究同 XRD 技术用于结晶材料的结构研究，其实就是用透 射方法在小角度范围内获取样品的衍射谱。因此适 用 SAXS 技术获取 XRD 图谱的样品, 其晶胞参数或 是结构周期一般都在纳米量级。换言之, 适用 SAXS 技术测量晶体结构或周期有序结构的样品体系, 通 常是由颗粒为最小散射单元组成的超结构, 而不是 由原子为最小散射单元组成的晶体结构, 尽管超结 构可能具有晶体结构一样的对称性和空间群。 $\mathrm{Li}$ 等 ${ }^{[48]}$ 以 $\mathrm{Fe}_{3} \mathrm{O}_{4} @ \mathrm{SiO}_{2}$ 磁性纳米棒为组装单元, 在该 纳米棒形成的浓聚悬浮液中构筑了周期性排列的胶 体晶体阵列(Crystalline Colloidal Array, CCA), 同步 辐射 SAXS 技术研究证实该胶体晶体具有不同寻常 的单斜晶体结构, 如图 9 所示。这种胶体晶体同时 以光子带隙和双折射现象的形式展现出光学各向异 性。这一研究表明, 上述胶体晶体作为一种光子晶 体, 使磁调控其结构色和透射率成为可能, 同时也 为理解胶体自组装提供了一个新的模型系统, 且为 磁光效应的多功能显示、防伪和智能窗口应用等各 种智能光学材料的应用开辟了新机会。

Zhang 等 ${ }^{[49]}$ 也报道了用 SAXS 等技术对胶体系 统的研究, 发现多种无机纳米颗粒均可在多种熔融 无机盐中稳定存在。SAXS 实验结果为这种稳定存 在提供了有力的实验证据, 如图 10 所示。理论分析
和分子动力学模拟进一步表明，正是因为表面结合 的一层溶剂离子在溶质粒子周围的熔盐中产生长程 电荷密度振荡, 从而阻止溶质粒子的聚集。这种对 于无机颗粒在无机盐中的胶状复合的研究为胶体科 学领域的发展和应用开辟了新的思路。

\section{3 谱学应用示例}

\subsubsection{XAFS 谱}

同步辐射 X 射线吸收精细结构(SR-XAFS)谱学 技术是用来研究原子近邻结构的重要实验手段, 主 要用于研究吸收原子的价态和配位结构(包括配位 原子数、种类、距离等)。XAFS 谱测量的是样品吸 收系数随入射 $\mathrm{X}$ 射线能量的变化关系，因此用于 XAFS 谱研究的实验装置必须具有能量连续可调的 $\mathrm{X}$ 射线源。毫无疑问同步辐射光源是 XAFS 技术的 最理想光源, 事实上也正是同步辐射光源的发展才 使得 XAFS 技术真正成为一种用于物质结构研究的 实用技术。由于同步辐射光源的广谱特性，其涵盖 的能量范围几乎覆盖元素周期表内所有元素的吸收 边。当然，依托同步辐射光源的 XAFS 谱线站因设 计目标及技术上的原因，各自有自己的适用能量范 围。不得不说的是几乎所有配置了双晶单色器(能量 分辨率在 $10^{-4}$ 量级)的同步辐射光束线都能用于 XAFS 谱的测试。另一方面，由于 XAFS 信号仅来 源于物质中的原子近邻结构, 并不要求研究对象 

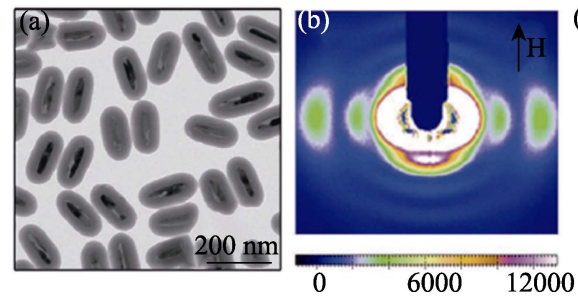

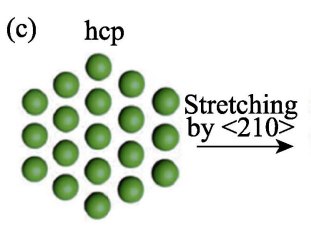

(001)

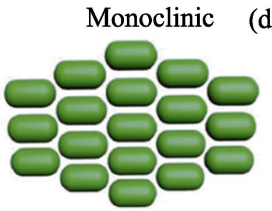

(010)
$\| i \theta=0^{\circ}$

musto $\uparrow H$

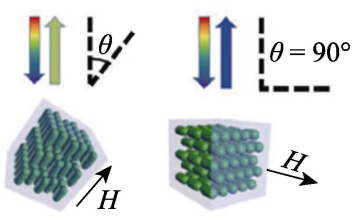

图 9 通过 $\mathrm{SAXS}$ 技术揭示的 $\mathrm{Fe}_{3} \mathrm{O}_{4} @ \mathrm{SiO}_{2}$ 纳米棒在磁场调控下的结构演化过程 ${ }^{[48]}$

Fig. 9 Structural evolution of $\mathrm{Fe}_{3} \mathrm{O}_{4} @ \mathrm{SiO}_{2}$ nanorods under magnetic field studied by $\mathrm{SAXS}^{[48]}$

(a) TEM image of the $\mathrm{Fe}_{3} \mathrm{O}_{4} @ \mathrm{SiO}_{2}$ nanorods; (b) Small-angle X-ray diffraction pattern of an aqueous suspension of $\mathrm{Fe}_{3} \mathrm{O}_{4} @ \mathrm{SiO}_{2}$ nanorods (volume fraction $\phi=21 \%$ ) in the presence of a 200-Gauss external magnetic field, the color bar on the bottom shows the relative diffraction intensity; (c) Schematic illustration of the stretching of the hep lattice along the $<210>$ direction. The (001) facet of the original hep lattice was transformed into the (010) facet of the new monoclinic structure. The main axis of nanorods is aligned along the $<201>$ direction of the monoclinic lattice, which is parallel to external magnetic fields; (d) Schematic illustration of magnetically tuning the Bragg diffractions from crystalline colloidal array by changing the angle $(\theta)$ between the magnetic field and the incident light

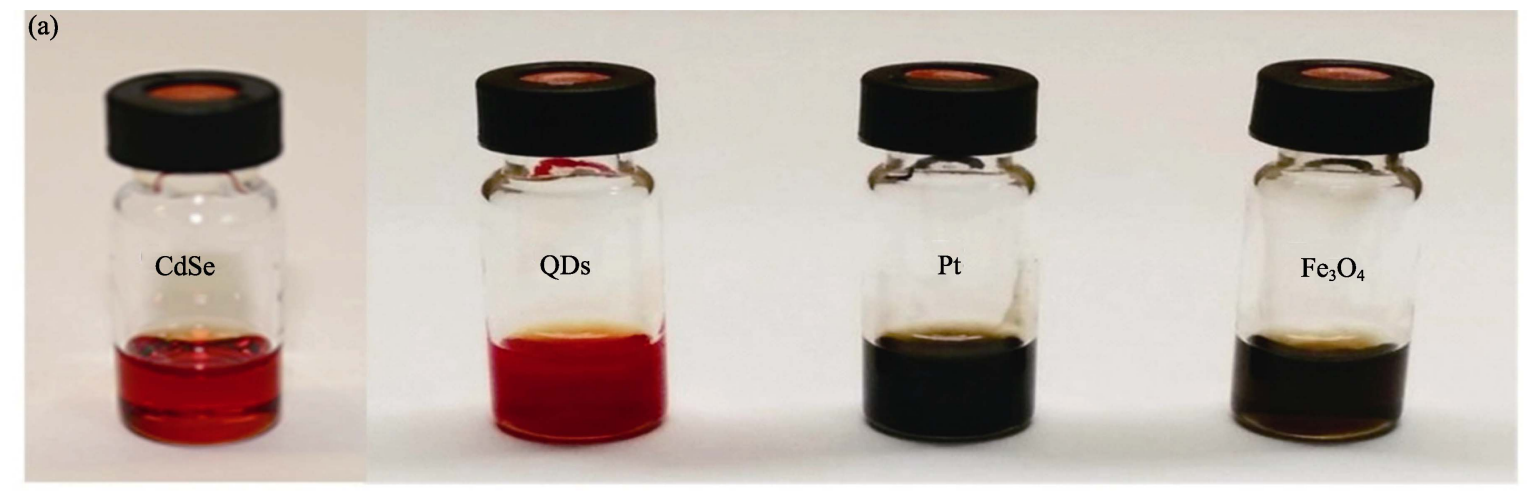

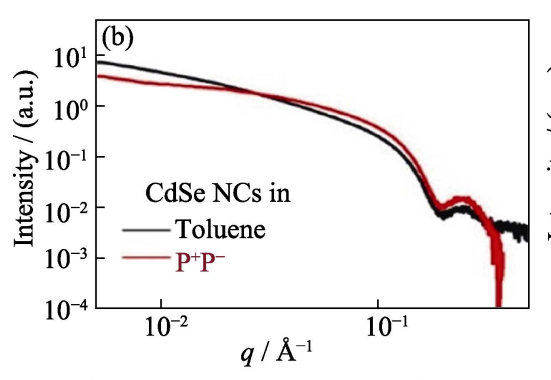
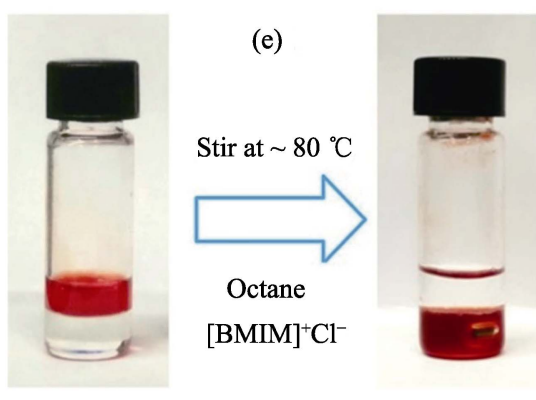
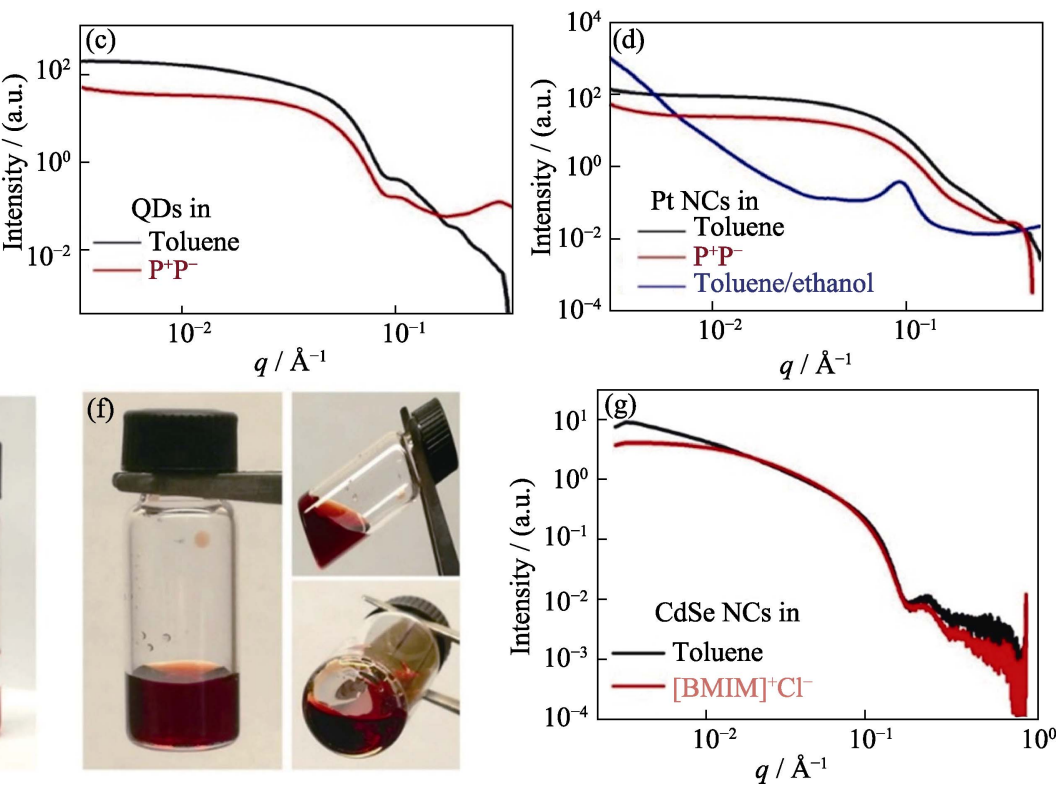

图 10 SAXS 技术为纳米颗粒在熔融无机盐中稳定存在提供的实验证据 ${ }^{[49]}$

Fig. 10 Experimental evidence for the stable presence of nanoparticles in molten inorganic salts provided by SAXS technique ${ }^{[49]}$ (a) Photographs of stable nanocrystals (NC) colloids in $\mathrm{P}^{+} \mathrm{P}^{-}$; (b-d) Small-angle X-ray scattering (SAXS) patterns of CdSe (b), quantum dots (QDs) (c) and Pt NCs (d) in toluene and $\mathrm{P}^{+} \mathrm{P}^{-}$. The SAXS patterns of NCs in $\mathrm{P}^{+} \mathrm{P}^{-}$resemble those of NCs in toluene, indicating high NC colloidal stability;

(e) Photographs showing the phase transfer of CdSe NCs from octane to $[\mathrm{BMIM}]^{+} \mathrm{Cl}^{-}$without additional ligands; (f) Photographs of stable CdSe NC colloids in [BMIM] ${ }^{+} \mathrm{Cl}^{-}$; (g) SAXS patterns of CdSe NCs in toluene (with OA/TOP/TOPO) and [BMIM] $]^{+} \mathrm{Cl}^{-}$.

All samples were gently heated to keep $[\mathrm{BMIM}]^{+} \mathrm{Cl}^{-}$in the molten state

$$
\text { (1 }=0.1 \mathrm{~nm})
$$

必须具备长程有序结构, 因此适用 XAFS 技术研究 其原子近邻结构的样品体系极其广泛。除单原子气 体外, 多原子气体、液体与固体、晶体与非晶体、
块体与粉末、薄膜与界面等各种形态的物质都适合 采用 XAFS 技术研究。要获取质量足够好的 XAFS 谱，除要求拟用的同步辐射光束线提供的 $\mathrm{X}$ 射线能 
量覆盖样品中待测元素吸收边的能量范围外, 还要 求样品厚度满足最佳信噪比的要求 $(\Delta \mu d \approx 1)$ 。由于 XAFS 技术的普适性, 可以说 XAFS 线站是同步辐 射装置上最繁忙的线站之一。用 XAFS 技术研究过 的无机材料数不胜数, 无机催化剂微观结构与机制的 研究工作在 XAFS 应用中就占有相当大的比例 ${ }^{[50-53]}$ 。

Cao 等 ${ }^{[54]}$ 利用原子层沉积 (Atomic Layer Deposition, ALD)技术，在 $\mathrm{SiO}_{2}$ 担载的 $\mathrm{Pt}$ 金属纳米 颗粒表面上精准构筑出原子级分散的 $\mathrm{Fe}_{1}(\mathrm{OH})_{x}$ 物种, 形成 $\mathrm{Fe}_{1}(\mathrm{OH})_{x}-\mathrm{Pt}$ 界面位点催化剂结构。 $\mathrm{Fe}_{1}(\mathrm{OH})_{x}-\mathrm{Pt}$ 界面位点容易与 $\mathrm{CO}$ 反应, 促进氧的活化。他们利 用原位 XAFS 技术证实 $\mathrm{Fe}_{1}(\mathrm{OH})_{x}$ 物种在催化反应气 氛中的结构是 $\mathrm{Fe}_{1}(\mathrm{OH})_{3}, \mathrm{Fe}_{1}$ 原子与 $\mathrm{Pt}$ 纳米颗粒表面 $\mathrm{Pt}$ 原子形成 $\mathrm{Fe}_{1}-\mathrm{Pt}$ 金属键，具有超高还原特性，并 确定在 Pt 颗粒表面上形成的 $\mathrm{Pt}-\mathrm{Fe}_{1}(\mathrm{OH})_{3}$ 界面位点是
其催化活性中心，揭示了其催化反应机理如图 11 所 示。这一研究为设计高活性金属催化剂提供了新方 法, 为解除氢燃料电池一氧化碳 “中毒休克” 危机、 延长电池寿命、拓宽电池使用温度等扫清了障碍。

无机 $\mathrm{NaFePO}_{4}$ 材料因高容量、低成本、高稳定 和无毒性等优良特性, 已成为钠离子电池正极的首 选材料。Xiong 等 ${ }^{[55]}$ 在 BSRF 的 4B7B 实验站采集 了轻元素 $\mathrm{Na}$ 和 $\mathrm{O}$ 的 XAFS 谱, 结合 XAFS 谱和拉 曼光谱的分析, 从原子尺度结构上揭示了非晶 $\mathrm{NaFePO}_{4}$ 电极的无序增强了其钠存储性能的本质。 研究结果表明共棱的 $\mathrm{FeO}_{6}$ 八面体经非晶化转变为 各种 $\mathrm{FeO}_{n}$ 多面体是钠离子电池获得优异性能的关 键，如图 12 所示。基于此研究，Xiong 等 ${ }^{[55]}$ 用机械 化学的方法成功调控了 $\mathrm{NaFePO}_{4}$ 正极材料的无序度, 改善了钠离子电池的电化学性能。
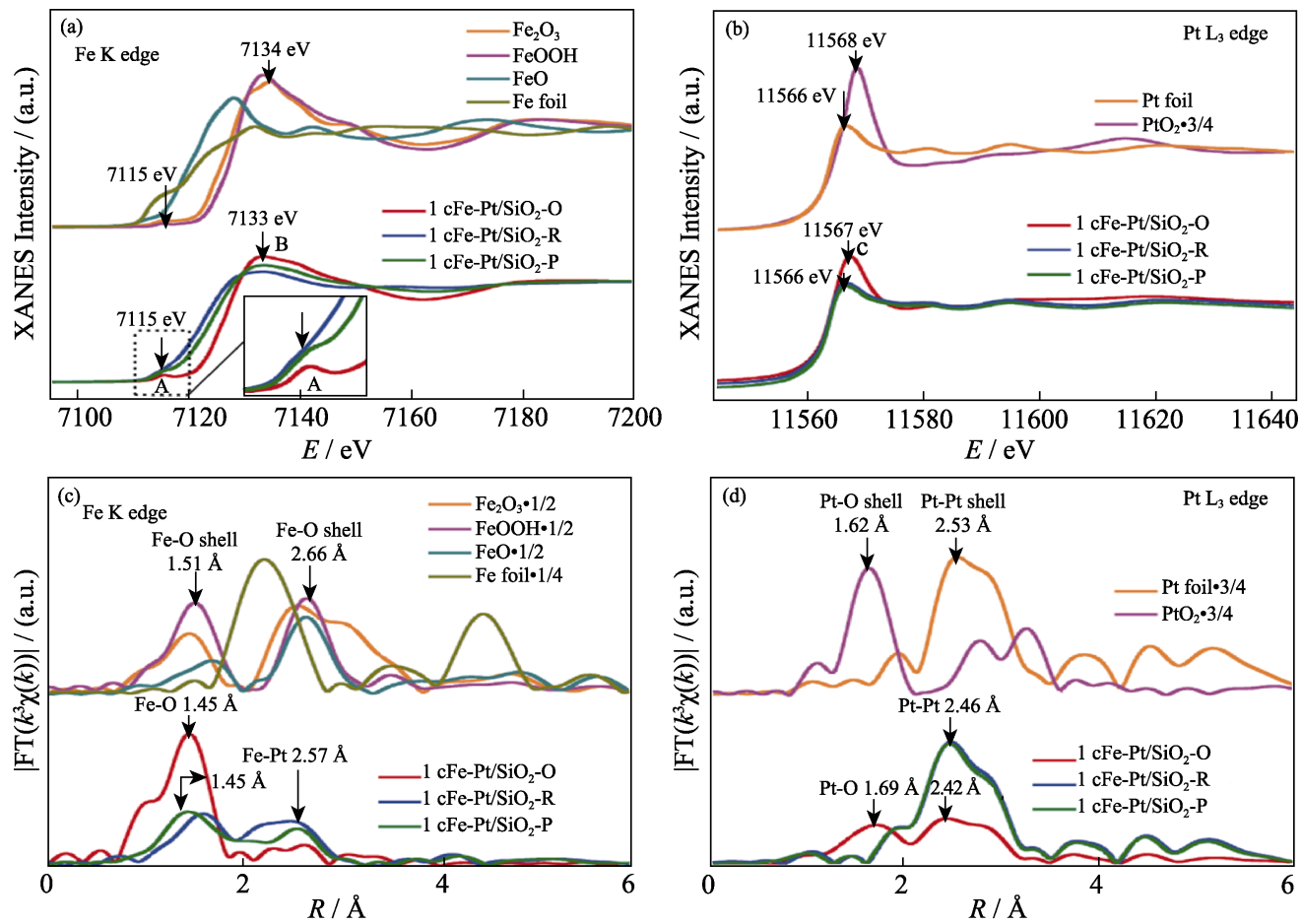

(e)

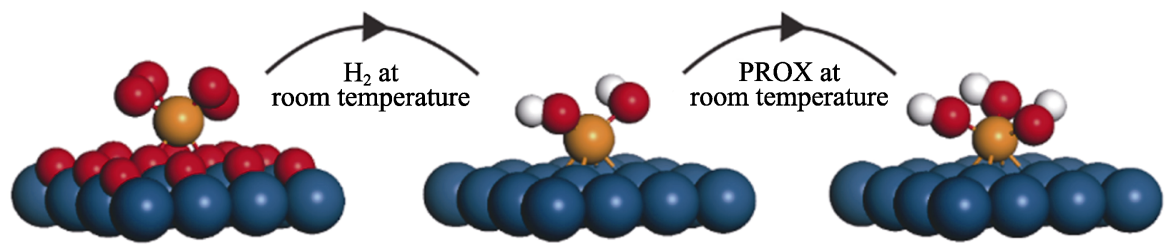

图 $11 \quad 1 \mathrm{cFe}-\mathrm{Pt} / \mathrm{SiO}_{2}$ 催化剂的原位 XAFS 测试及其催化机理示意图 ${ }^{[54]}$

Fig. 11 In situ XAFS measurements of the $1 \mathrm{cFe}-\mathrm{Pt} / \mathrm{SiO}_{2}$ catalyst and its schematic illustration of catalytic mechanism ${ }^{[54]}$

(a-d) XANES spectra at Fe K edge (a) and $\mathrm{Pt}_{2}$ edge (b), detailed structural information shown in the $k^{3}$-weighted FT spectra in R-space at the $\mathrm{Fe} \mathrm{K}$ edge (c) and the $\mathrm{Pt} \mathrm{L}_{3}$ edge (d) ( $E$ : energy, $R$ : distance between absorbing atom and neighbouring scatterer atoms, without correcting for scattering phase shift, and $\chi(k)$ : the amplitude of the EXAFS oscillations as a function of photoelectron wavenumber

$k$ (a.u., arbitrary units). The inset in (a) shows a magnified view of the pre-edge peak A. The energy positions of Fe pre-edge peak A, Fe white-line peak B and Pt white-line peak C, as well as R-space peak positions of the shells of Fe-O, Fe-Fe, Fe-Pt, Pt-O and Pt-Pt, are also shown; (e) Schematic models of $1 \mathrm{cFe}-\mathrm{Pt} / \mathrm{SiO}_{2}-\mathrm{O}$ (left), $1 \mathrm{cFe}-\mathrm{Pt} / \mathrm{SiO}_{2}-\mathrm{R}$ (middle) and $1 \mathrm{cFe}-\mathrm{Pt} / \mathrm{SiO}_{2}-\mathrm{P}$ (right),

$\mathrm{Fe}, \mathrm{O}, \mathrm{H}$ and $\mathrm{Pt}$ atoms are shown in orange, red, white, and blue, respectively $(1 \AA=0.1 \mathrm{~nm})$ (colorful figures are available on website) 

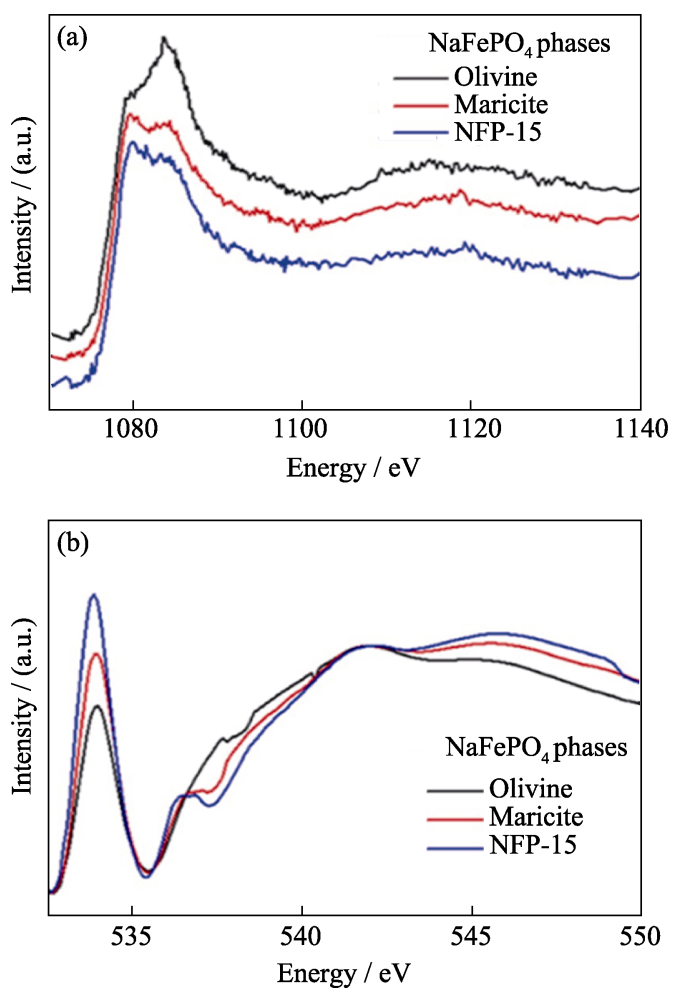

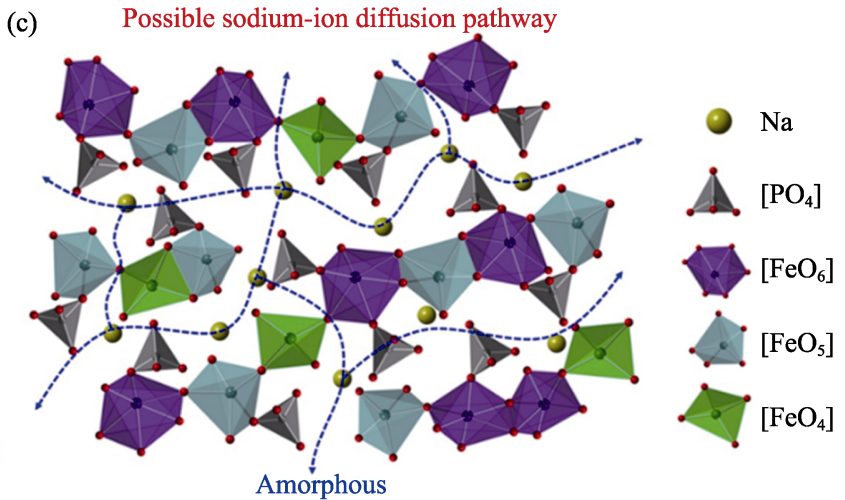

(d)

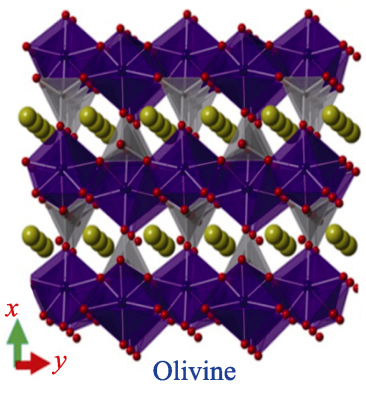

(e)

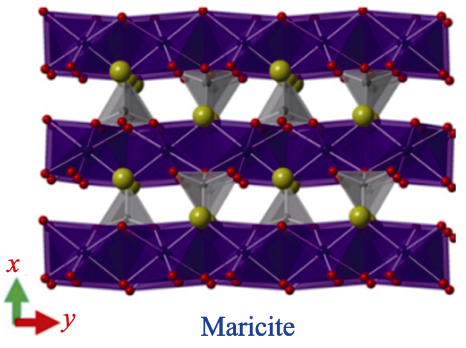

图 $12 \mathrm{NaFePO}_{4}$ 的 XAFS 光谱及 $\mathrm{Na}$ 扩散机制示意图 ${ }^{[55]}$

Fig. 12 XAFS spectra of $\mathrm{NaFePO}_{4}$ and schematic illustrations for $\mathrm{Na}$ diffusive mechanism ${ }^{[55]}$

X-ray absorption near edge structure spectra of Na K-edge (a), O K-edge (b) for olivine-type, maricite-type and polymorphic composites obtained by milling for $15 \mathrm{~h}$ (NFP-15); Schematic illustrations for Na diffusive mechanism: possible sodium-ion diffusive pathways and atomistic structure for amorphous $\mathrm{NaFePO}_{4}$ (c), structural sketching diagrams of olivine-type $\mathrm{NaFePO}_{4}$ (d), and maricite-type $\mathrm{NaFePO}_{4}(\mathrm{e})$ Colorful figures are available on website

$\mathrm{Li}$ 等 ${ }^{[56]}$ 利用 XAFS 技术研究了锂离子电池的阴 离子氧化还原反应和活化机理, 证实了 $\mathrm{Fe}^{3+}$ 替代是 触发立方 $\mathrm{Li}_{2} \mathrm{TiO}_{3}$ 电化学活性和氧化还原活性的原 因, 这项工作对于利用阴离子氧化还原过程设计大

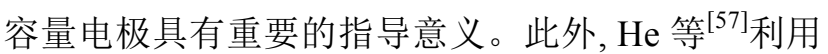
硫化锡 $(\mathrm{SnS})$ 的多个能带随着温度的演变规律, 通过 引入 $\mathrm{Se}$ 优化调控有效质量和迁移率之间的矛盾, 并 在储量丰富、成本低廉、环境友好的 $\mathrm{SnS}$ 晶体材料 中实现了高的热电性能。该研究首先获得了原位变 温 $\mathrm{SnS}$ 材料的精确晶胞参数, 再从 XAFS 数据中得 到目标原子的原子占位信息, 如图 13 所示。结合 XRD 和 XAFS 结果, 该研究揭示了 $\mathrm{SnS}$ 无机材料的 晶体结构及物理输运机理。操纵能带的策略也为优 化热电材料性能提供了一种新的途径。

\subsubsection{XPS 谱}

无机材料的宏观性质 (如电学性能、热力学性 能、磁学性能、光学性能等)均与其材料本身的原子 结构和电子结构有关 ${ }^{[58]}$, 研究材料的宏观特性必然 要从其原子结构和电子结构出发。 X 射线光电子能 谱(X-ray Photoelectron Spectroscopy, XPS)是研究物 质电子结构最为直接的实验手段, 可以获得从原子 深层的芯能级到浅层价带的全波段电子信息。由于 材料电子结构自身的复杂性以及关联电子之间相互
作用结果的精细性, XPS 对光源的光通量和能量分 辨率有较高的要求。虽然基于常规光源的 XPS 实验 设备已得到广泛应用, 但在光源能谱的连续性和光 源品质上对获取材料精细的电子结构信息越来越难 以满足需求。基于同步辐射光源的 $\mathrm{X}$ 射线光电子能 谱(SR-XPS)以其优异的特性已成为无机材料领域研 究电子结构的必备工具。中国大陆目前的三个同步 辐射光源都可开展 XPS 实验研究。由于电子在物质 中的逃逸深度有限, XPS 更适用于在真空环境下研 究材料的表面和界面信息。因而, 二维材料的结构 稳定性、电子能带结构、界面反应和拓扑特性等是 XPS 技术的主要研究对象。研究薄膜电子结构及薄 膜原子与无机祄底间界面反应的首选方法就是 XPS, 典型的二维材料有无机祄底上生长的单原子层薄 膜、石墨烯等。

已有的研究预测在 $\mathrm{Bi}_{2} \mathrm{Te}_{3}(111)$ 基底上用分子束 外延技术生长的类似于石墨烯的锡烯是一种二维拓 扑绝缘体。李金梅等 ${ }^{[59]}$ 用高分辨的同步辐射光电子 能谱技术研究了在 $\mathrm{Bi}_{2} \mathrm{Te}_{3}$ 祄底上生长的 $\mathrm{Sn}$ 薄膜的 电子结构。锡烯的各向异性电子能带结构可以解释 其各向异性的电子性质和输运行为。通过研究 $\mathrm{Sn} / \mathrm{Bi}_{2} \mathrm{Te}_{3}$ 系统的界面反应, 得到了新颖的物理结果, 如图 14 所示。研究发现 $\mathrm{Sn}$ 与 $\mathrm{Bi}_{2} \mathrm{Te}_{3}$ 之间的界面相 

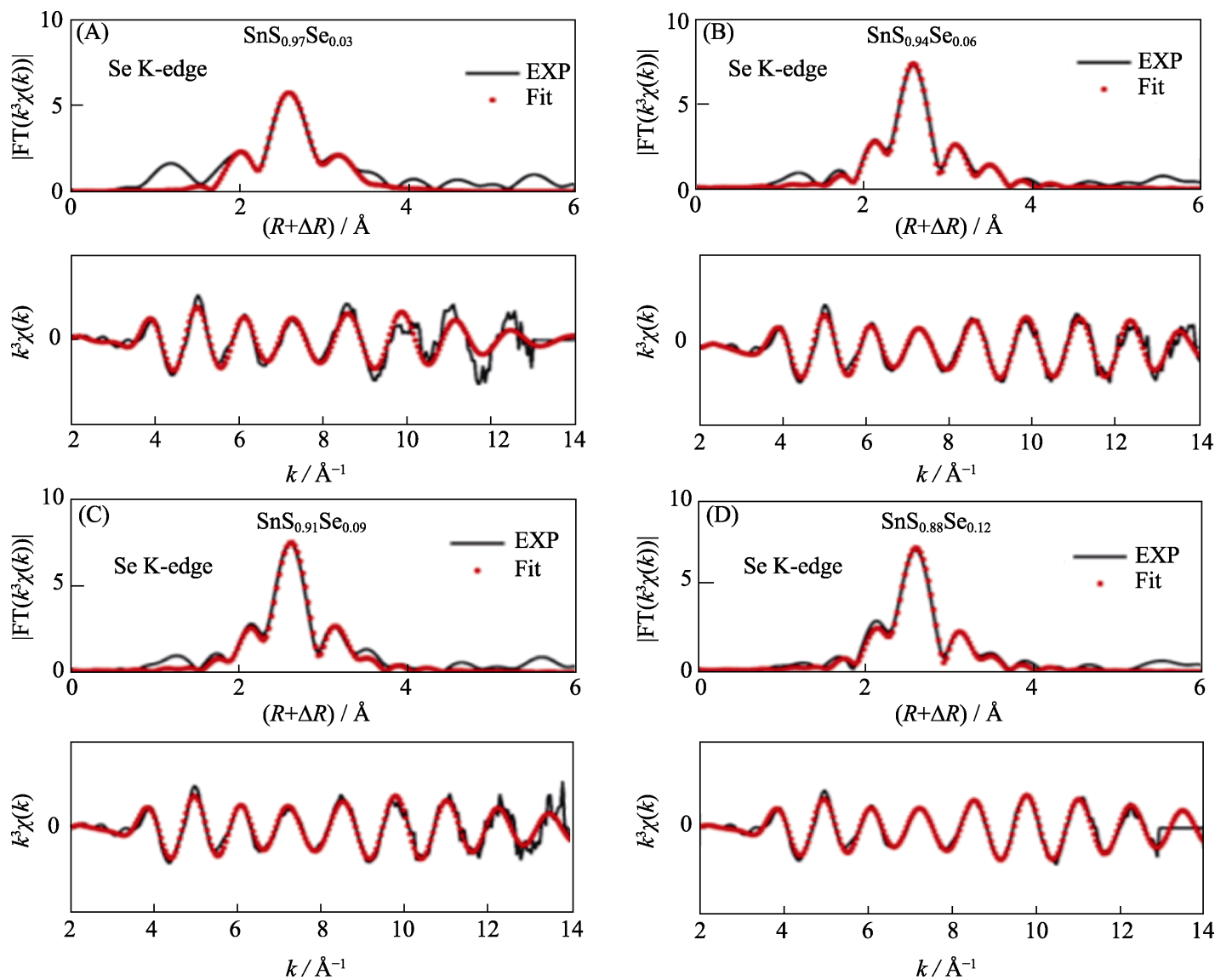

图 $13 \mathrm{SnS}_{1-x} \mathrm{Se}_{x}$ 的 $\mathrm{K}$ 边 $k^{3}$ 权重 $\mathrm{EXAFS}$ 振荡的傅里叶变换光谱图及其拟合结果 ${ }^{[57]}$

Fig. 13 Fitting of the Fourier transforms of $k^{3}$ weighted EXAFS oscillations at Se K-edge of $\mathrm{SnS}_{1-x} \mathrm{Se}_{x}{ }^{[57]}$

(A) $x=0.03$; (B) $x=0.06$; (C) $x=0.09$; (D) $x=0.12$

( $1 \AA=0.1 \mathrm{~nm})$ (colorful figures are available on website)

(a)

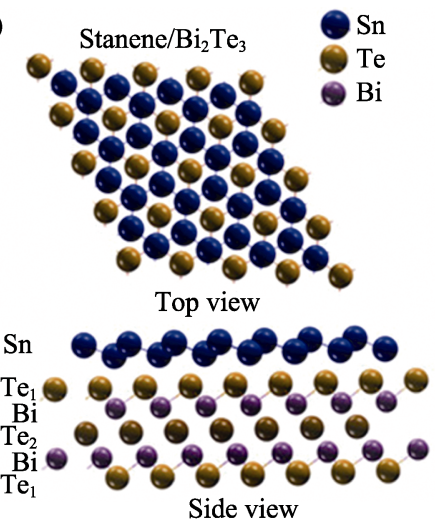

(b)
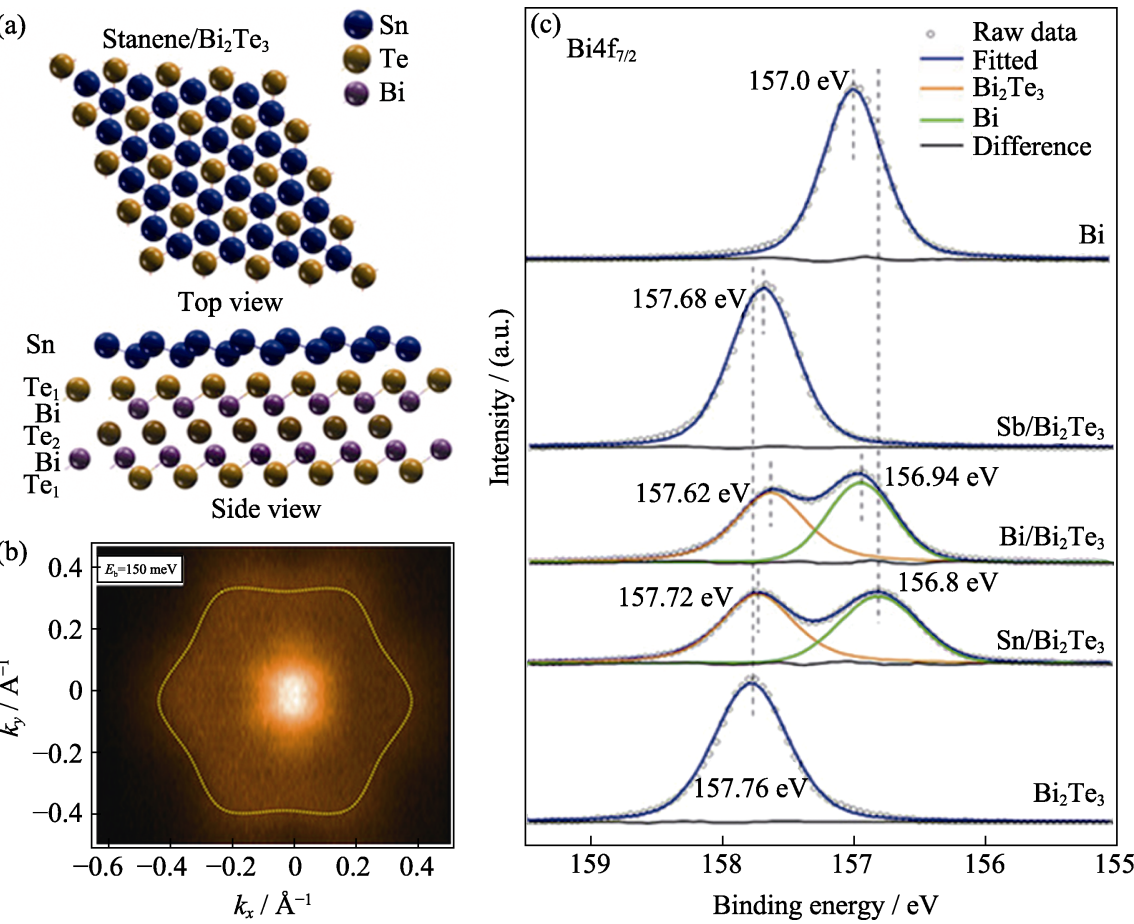

图 14 SR-XPS 实验研究的二维 $\mathrm{Sn} / \mathrm{Bi}_{2} \mathrm{Te}_{3}$ 材料的电子结构和界面相互作用 ${ }^{[59]}$

Fig. 14 Electronic structure and interfacial interaction of $2 \mathrm{D} \mathrm{Sn} / \mathrm{Bi}_{2} \mathrm{Te}_{3}$ material studied by SR-XRS ${ }^{[59]}$

(a) Perspective top and side views of atomic structure of stanene/ $\mathrm{Bi}_{2} \mathrm{Te}_{3}$; (b) ARPES intensity evolution of CE contours in the 2D Brillouin zone at the specified binding energies; (c) XPS results of $\mathrm{Bi}_{4} \mathrm{f}_{7 / 2}$ core levels. The $\mathrm{Bi} 4 \mathrm{f}_{7 / 2}$ spectra are measured at $500 \mathrm{eV}$ under room temperature $(1 \AA=0.1 \mathrm{~nm})$ (colorful figures are available on website) 
互作用不是以前报道的范德华力, 而是一种界面化 学反应形成的 $\mathrm{Sn}-\mathrm{Te}$ 键合。这一研究表明必须重新 分析 $\mathrm{Bi}_{2} \mathrm{Te}_{3}$ 祄底上锡烯的形成机制, 这也为该种 二维材料的制备和特性研究提供了重要思路。

热电材料主要通过泽贝克效应和佩尔捷帖效应 实现废热发电和电能制冷, 是一种使热能和电能相 互转换的绿色能源功能材料。热电材料应用的主要 瓶颈是其 $Z T$ 值很难突破 1 , 然而已有的一项研究发 现无机 $\mathrm{SnSe}$ 单晶材料的高温 $Z T$ 值达到了 2.6。然 而, 对于 $\mathrm{SnSe}$ 的电子结构和最重要的空穴掺杂机 制还缺乏全面的了解, 理解该材料中高 $Z T$ 值的机 理也具有重要的应用价值。Wang 等 ${ }^{[60]}$ 利用极低温 量子输运测量和角分辨光电子能谱(Angle Resolved Photoelectron Spectroscope, ARPES) 实验首次揭示 了硒化锡的特殊输运性质, 如图 15 所示。研究发现 具有准线性能量色散的独特布丁型价带, 证明 $\mathrm{SnSe}$ 中的 $\mathrm{p}$ 型掺杂是由局部 $\mathrm{SnSe}_{2}$ 微畴相偏析通过界面 电荷转移控制的。这一研究为进一步改进具有高效
能的热电材料提供了重要依据。

\subsubsection{VUV 谱}

真空紫外(VUV)光谱技术可用来研究无机材料 (无机光催化剂、无机发光材料、激光材料等)的发 光特性。常规的真空紫外光谱技术的波长单一, 且 VUV 激光调谐范围较窄。相比之下, 基于同步辐射 光源的 VUV 光谱技术准直性好、波段连续可调、 亮度高且方便开展原位和时间分辨实验, 在材料研 究中得到越来越广泛的应用。

一个典型的 VUV 光谱技术应用实例是荧光粉体材 料 $\mathrm{CaSi}_{10-n} \mathrm{Al}_{2+n} \mathrm{O}_{n} \mathrm{~N}_{16-n}: x \mathrm{Eu}^{2+}(m=2, n=0 \sim 1, x=0.1 \% \sim 8 \%)$ 发光特性的研究, 如图 16 所示。Wang 等 ${ }^{[61]}$ 利用 VUV-UV 激发谱对 $\mathrm{CaSi}_{10-n} \mathrm{Al}_{2+n} \mathrm{O}_{n} \mathrm{~N}_{16-n}: x \mathrm{Eu}^{2+}$ 材料发 光特性的实验研究表明: 在 $254 \mathrm{~nm}$ 的紫外线激发 下，该材料可持续发出黄光，并且持续时间与 $\mathrm{Eu}^{2+}$ 和氧气的浓度密切相关。当 $n=0, x=0.5 \%$ 时, 发光持 续时间可达 $60 \mathrm{~min}$ 。这一研究揭示了电子作为载流 子在紫外线激发过程中直接从 $\mathrm{Eu}^{2+}$ 的 $4 \mathrm{f}$ 基态激发到
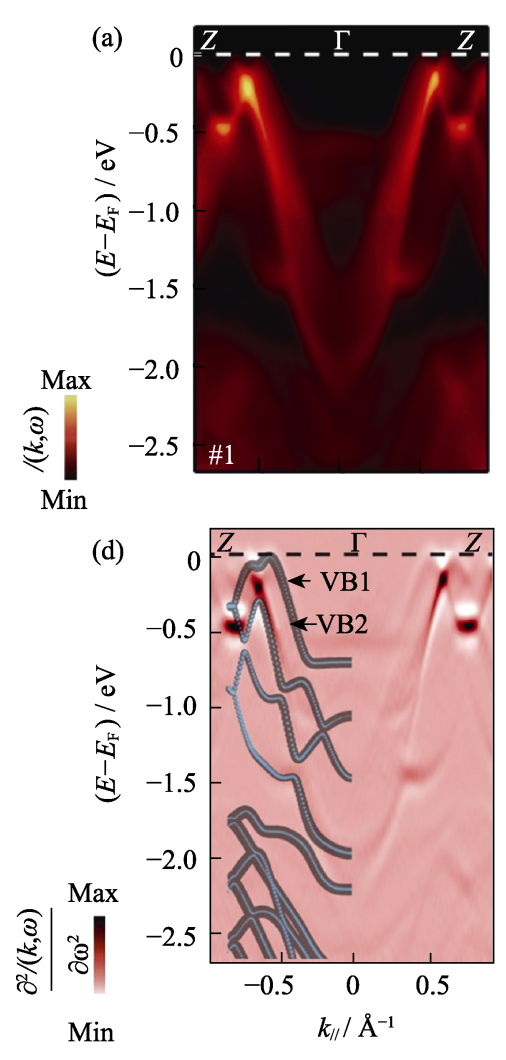
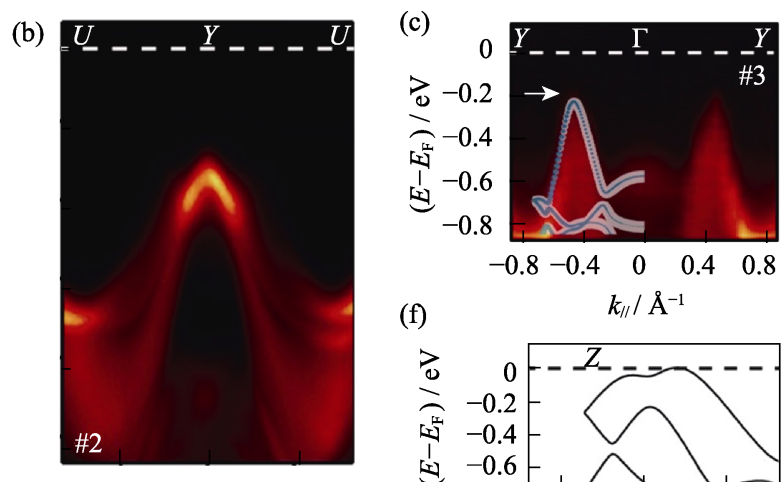

(f)

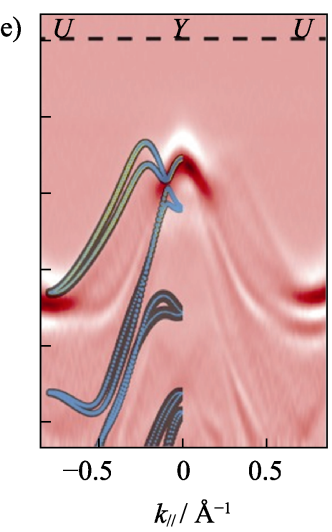

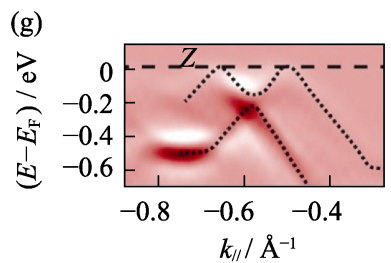

(h)

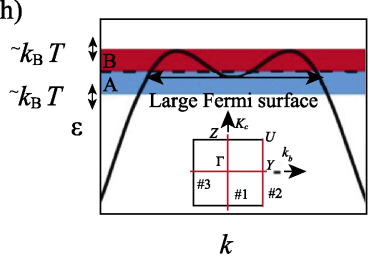

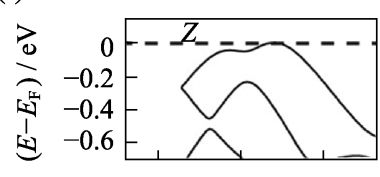

图 15 ARPES 揭示的 $\mathrm{p}$-SnSe 的电子结构 ${ }^{[60]}$

Fig. 15 Electronic structure of p-SnSe resolved by ARPES ${ }^{[60]}$

ARPES measures band dispersions along high-symmetry directions of $Z-\Gamma-Z(\mathrm{a}), U-Y-U$ (b), and $Y-\Gamma-Y$ (c), taken with $50 \mathrm{eV}$ photon energy. The second derivative plots (d) and (e), corresponding to (a) and (b), respectively, directly compared with the DFT calculations, in which the weight of $\mathrm{Sn} 5 \mathrm{~s}$ and Se4p orbitals are represented by light blue and yellow, respectively; Close-up of the top VBs along $\Gamma-Z$ reveals pronounced differences between the theoretical band dispersion (f) and the ARPES measured results (g), as highlighted by the X-shaped dashed lines;

(h) Schematic plot of a pudding-moul shaped VB with corrugations, which leads to giant $S$ due to the band geometry effect with inset showing the ARPES cut directions in the projected two-dimensional first Brillouin zone (BZ) 

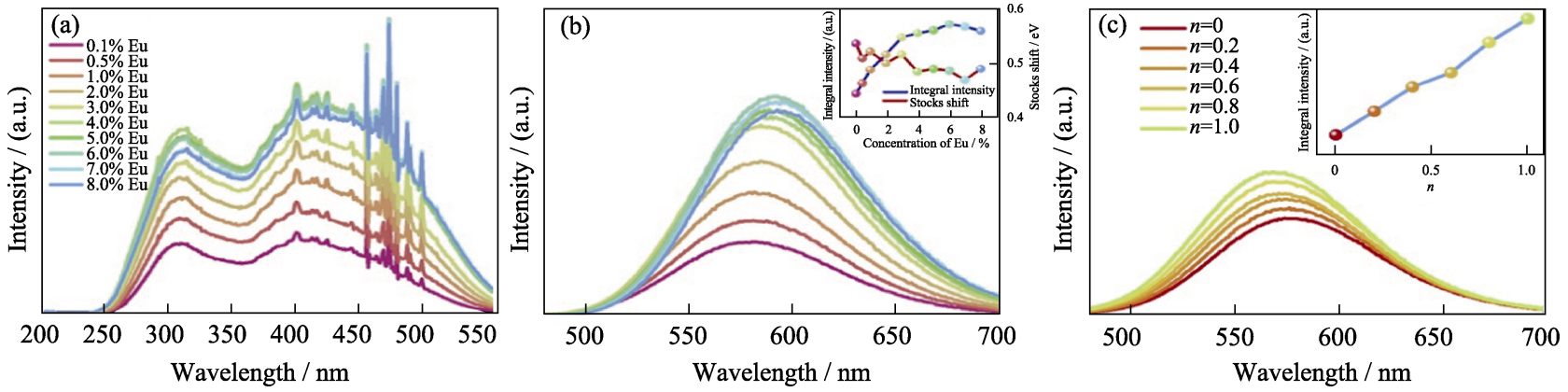

图 16 VUV-UV 光谱研究的 $\mathrm{CaSi}_{10-n} \mathrm{Al}_{2+n} \mathrm{O}_{n} \mathrm{~N}_{16-n}: x \mathrm{Eu}^{2+}$ 材料的持续发光特性 [61]

Fig. 16 Persistent luminescence properties of $\mathrm{CaSi}_{10-n} \mathrm{Al}_{2+n} \mathrm{O}_{n} \mathrm{~N}_{16-n}: x \mathrm{Eu}^{2+}$ material studied by VUV-UV spectra ${ }^{[61]}$

(a) Excitation and (b) emission spectra of $\mathrm{CaSi}_{10} \mathrm{Al}_{2} \mathrm{~N}_{16}: x \mathrm{Eu}(x=0.1 \%-8 \%)$ samples with inset showing the integral intensity (blue curve) and the Stokes shift (red curve) of those samples against Eu concentration (the sharp peaks in the range of 400-500 nm being caused by the Xe lamp equipped in the FLS 920 spectrophotometer); (c) Emission spectra of $\mathrm{CaSi}_{10-n} \mathrm{Al}_{2+n} \mathrm{O}_{n} \mathrm{~N}_{16-n}: 0.5 \% \mathrm{Eu}(n=0-1)$ samples with inset showing the integral intensity versus $n$ values (colorful figures are available on websites)

导带(Conduction Band, CB), 相关材料在防伪和信 息存储方面具有潜在的应用价值。

\section{4 成像应用示例}

近年来，同步辐射 X 射线成像(Synchrotron radiation-X-ray Imaging, SR-XRI)技术得到了飞速发 展。在空间分辨率上, 硬 $\mathrm{X}$ 射线波段已经实现优于 $30 \mathrm{~nm}$ 三维空间分辨率的同步辐射纳米成像, 软 $\mathrm{X}$ 射线波段的纳米成像空间分辨率也提高到 $10 \mathrm{~nm}$ 的 水平，相干衍射成像甚至可实现 $\mathrm{X}$ 射线波长量级的 空间分辨率。在实验技术上，各种基于同步辐射光 源的 $\mathrm{X}$ 射线成像方法 ${ }^{[63]}$ 相继被提出并得到发展完善, 如 X 射线显微 CT 成像(Micro-CT)、基于波带片的纳 米分辨全场成像 (Transmission X-ray microscopy, $\mathrm{TXM})$ 、纳米分辨探针扫描成像(Nano-probe)、相干衍 射成像(Coherent diffraction imaging, CDI)和几何放大 投影成像(Projection microscopy)等。中国大陆现有 的三个同步辐射装置都能开展 $\mathrm{X}$ 射线成像实验研 究。值得一提的是, 同步辐射纳米成像技术已由早 期的单一结构成像方法, 逐步发展出高分辨率、多 尺度、三维结构、元素分布、化学成分、价态分析 以及动态研究等多种结构信息的研究手段。同步辐 射 $X$ 射线成像技术与 $X$ 射线谱学技术相结合而发展 出来的谱学成像方法就是一个典型实例。 $\mathrm{X}$ 射线成像 相关的先进实验技术已被广泛应用于无机材料领域 的前沿研究中, 特别是应用于具有复杂形貌的特殊 功能材料 ${ }^{[64]}$, 以及热门的能源材料 ${ }^{[65]}$ 研究中。

$\mathrm{Qu}$ 等 ${ }^{[66]}$ 用水热法分别合成了花状和海胆状的 硅酸锌纳米颗粒。花状的硅酸锌纳米颗粒(锌硅石, $\left.\mathrm{Zn}_{3} \mathrm{Si}_{4} \mathrm{O}_{10}(\mathrm{OH})_{2} \cdot n \mathrm{H}_{2} \mathrm{O}\right)$ 具有新颖的黏土状层状结构, 而海胆状的硅酸锌(菱锌矿, $\mathrm{Zn}_{4} \mathrm{Si}_{2} \mathrm{O}_{7}(\mathrm{OH})_{2} \cdot \mathrm{H}_{2} \mathrm{O}$ ) 纳 米颗粒具有高度结晶的锌矿石结构。利用 $\mathrm{X}$ 射线纳 米成像技术研究了海胆状硅酸锌催化剂颗粒的结构
和形貌，结果提供了该颗粒内部其他方法难以观测 的结构细节, 如一些棒状粒子不是以径向的方式组 装到海胆状颗粒内部或者一些棒状粒子悬挂在规则 的径向组装的其他棒状粒子之间。这一研究揭示了 海胆状颗粒自组装过程中的结构缺陷, 如图 17 所示, 也为解释此种颗粒的形成机制和更好地发挥多孔状 材料的特性奠定了研究基础。

在锂离子电池材料方面, 具有高比容量、长循 环寿命、低毒性和廉价的镍钴锰三元电极材料以及
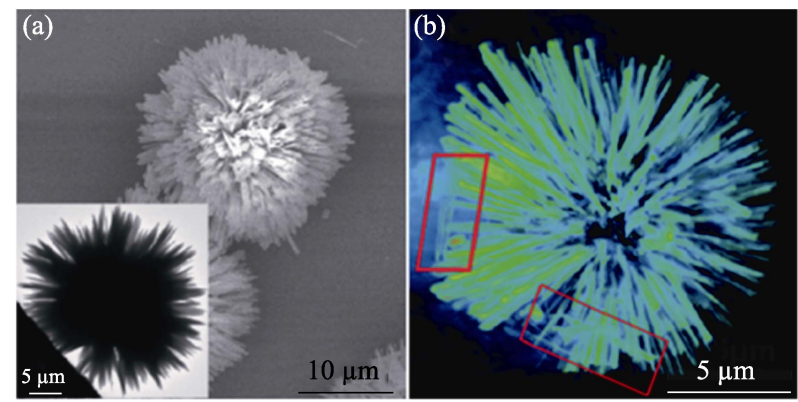

(c)
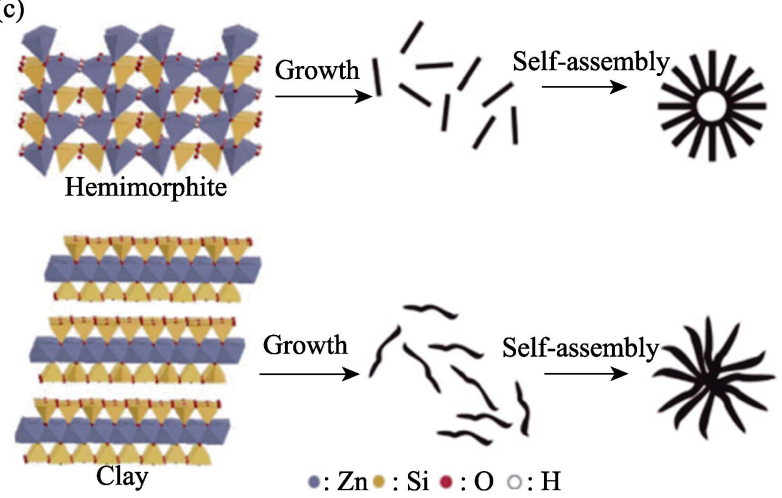

图 17 同步辐射 $\mathrm{X}$ 射线纳米成像技术研究的 $\mathrm{Zn}_{4} \mathrm{Si}_{2} \mathrm{O}_{7}(\mathrm{OH})_{2} \cdot \mathrm{H}_{2} \mathrm{O}$ 颗粒的结构缺陷和形成过程 ${ }^{[66]}$

Fig. 17 Structural defects and formation process of $\mathrm{Zn}_{4} \mathrm{Si}_{2} \mathrm{O}_{7}(\mathrm{OH})_{2} \cdot \mathrm{H}_{2} \mathrm{O}$ particles studied by SR-XRI ${ }^{[66]}$

(a) SEM image (inset: TEM image); (b) Reconstructed three dimensional rendering view of the urchin-like zinc silicate; (c) Schematic illustration of the preparation of the zinc silicate nanomaterials 
钴酸锂、锰酸锂、镍酸锂、磷酸铁锂及锂钴锰镍复 合氧化物电极材料一直是研究的热点。准确测定电 池电极材料中各元素含量及其空间分布对于提高电 池的能量密度和循环性能, 延长电池寿命, 以及优 化设计高性能电池电极材料等都具有十分重要的意 义。 $\mathrm{LiNi}_{1-x-y} \mathrm{Mn}_{x} \mathrm{Co}_{y} \mathrm{O}_{2}$ 是一种技术上重要的阴极材 料, 但在各种运行条件下, 特别是在富镍成分中, 通常会发生从层状到岩盐结构的表面重构。这种现 象导致其高压循环性能变差, 阻碍了其能量密度的 提高。Lin 等 ${ }^{[67]}$ 利用 $\mathrm{X}$ 射线断层成像技术研究发现: 多级结构的 $\mathrm{LiNi}_{0.4} \mathrm{Mn}_{0.4} \mathrm{Co}_{0.2} \mathrm{O}_{2}$ 球形颗粒表面出现 具有贫 $\mathrm{Ni}$ 富 $\mathrm{Mn}$ 的局部元素偏析现象, 如图 18 所 示。这一研究揭示了颗粒表面贫 $\mathrm{Ni}$ 富 $\mathrm{Mn}$ 的成分分 布是造成这个材料循环性能提高的原因, 也为以后 电池材料性能的进一步提高奠定了基础。此外, Wei 等 ${ }^{[68]}$ 也对 $\mathrm{LiNi}_{1-x-y} \mathrm{Mn}_{x} \mathrm{Co}_{y} \mathrm{O}_{2}$ 电极材料进行了纳米尺 度的 $\mathrm{X}$ 射线谱学成像技术研究。他们利用在不同能 量下采集的一系列图像上对应像素点的灰度值获得 基于该像素的吸收谱曲线, 从而获取元素成分及价 态的空间分布信息, 如图 19 所示。这一研究有助于 人们理解电池优异性能的物理机制。

\section{4 总结与展望}

同步辐射装置是研究包括无机材料在内的各种 物质结构的先进科研利器。基于同步辐射光源的各 种物质结构表征技术广泛应用于物理、化学、生物、 材料、地质、农林、医药、环保等各种学科领域, 贯 穿于样品制备、服役、失效等 “生命” 周期的各个 环节, 为揭示物质结构与性能的构效关系提供了第 一手的结构信息, 产生了许多重要的科学成果。本 文仅就常见的同步辐射实验技术在无机材料研究中 的典型应用进行了简要的介绍，但同步辐射实验技 术并不局限于文中列举这些技术, 在无机材料方面 的应用也不胜枚举, 文中给出的少许应用实例也不 过是挂一漏万。

同步辐射光源还将进一步发展完善。一方面, 同步辐射作为大科学装置, 其造价和运行经费都很 高，需要国家投资才能支持，当今世界同步辐射装 置数量少、用户群体多, 就目前国内现状而言, 我国 同步辐射装置所提供的机时还远不能够满足科研用 户的需求，同步辐射用户的发展壮大和科学技术的 需求，促进了同步辐射光源的发展；另一方面，

(a)

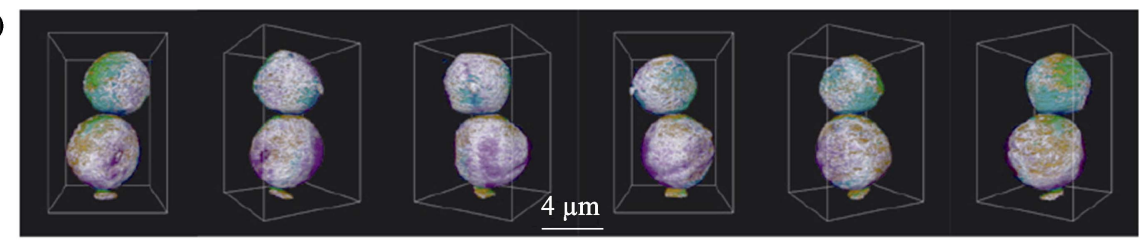

(b)

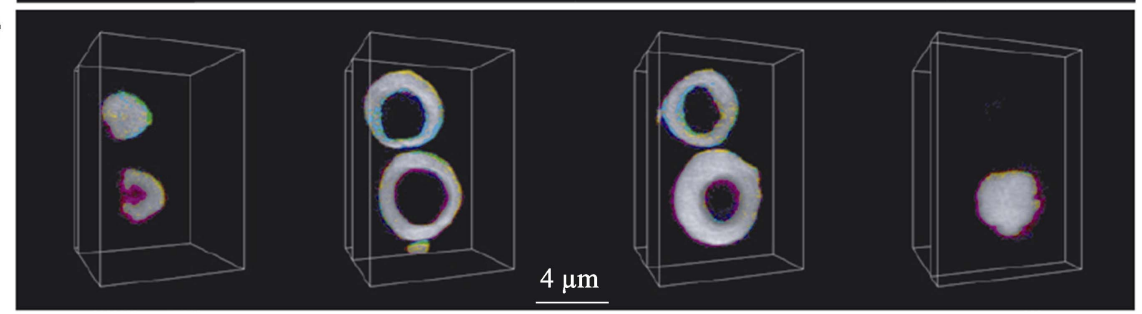

(c)

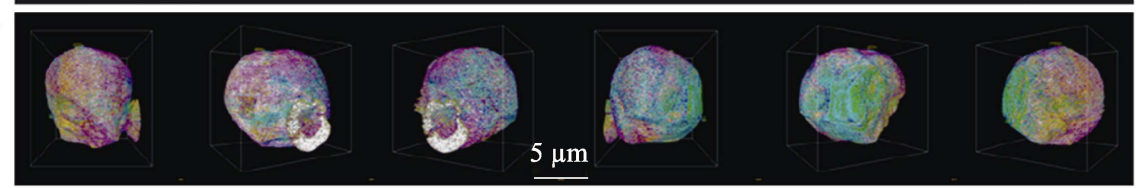

(d)

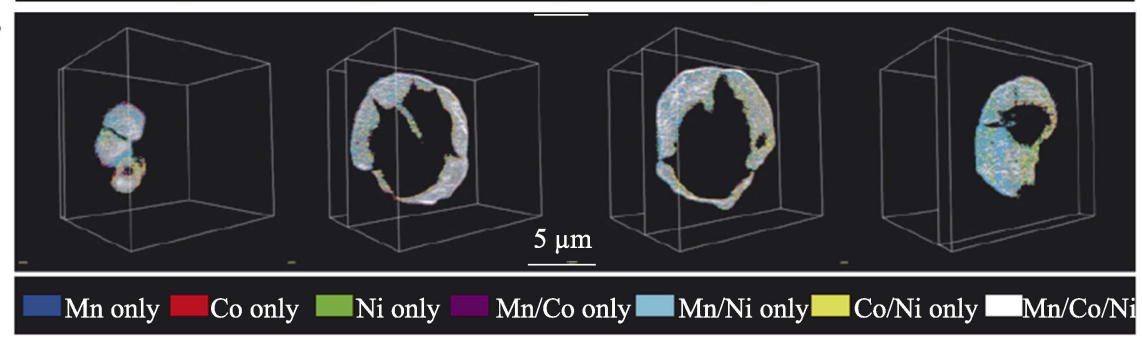

图 $18 \mathrm{X}$ 射线断层成像技术解析的颗粒三维元素分布 ${ }^{[67]}$

Fig. 18 3D element distribution of particles analyzed by X-ray tomography technique ${ }^{[67]}$

Elemental association maps of the as-made NMC precursor after spray pyrolysis (a,b) and the powder after annealing at $850{ }^{\circ} \mathrm{C}(\mathrm{c}, \mathrm{d})$;

$3 \mathrm{D}$ rendering of the elemental associations viewing the particles at different angles $(\mathrm{a}, \mathrm{c}) ; 2 \mathrm{D}$ slices of the elemental associations

cut through at different depths of the imaged particles $(b, d)$. The colours representing the elemental associations are shown at the bottom 


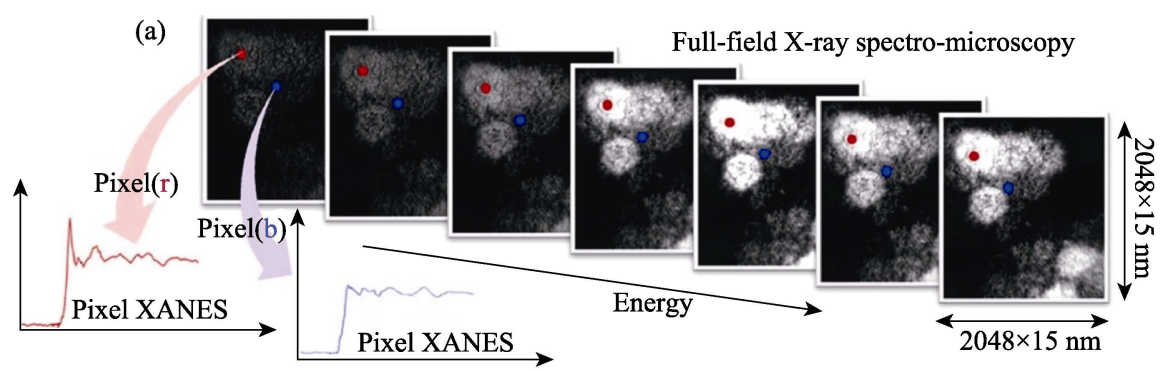

(b) Prior knowledge: known principle chemical species
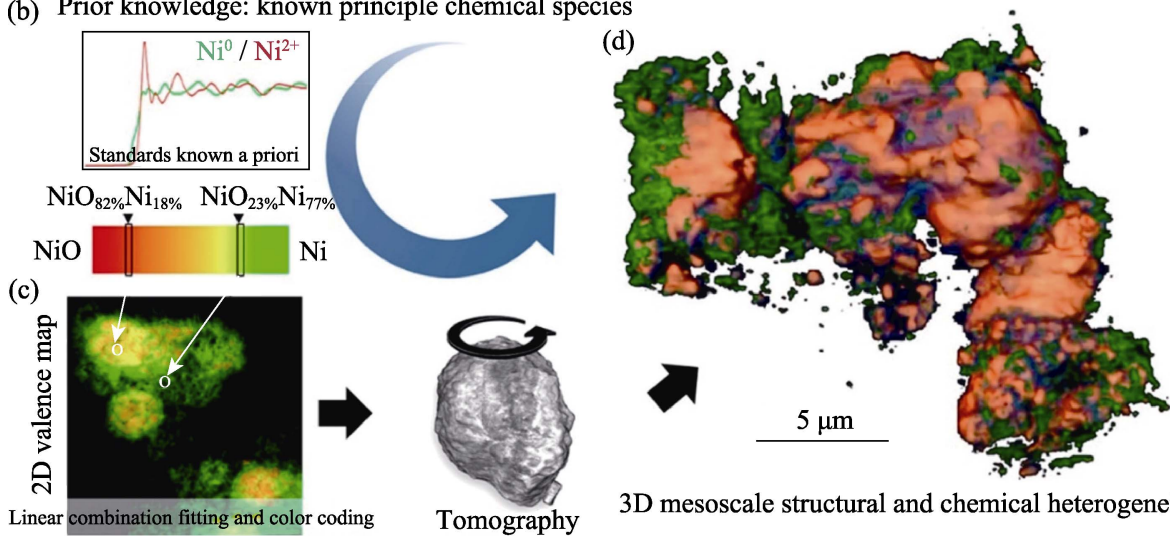

3D mesoscale structural and chemical heterogeneity

图 $19 \mathrm{X}$ 射线谱学成像技术研究的 $\mathrm{LiNi}_{0.4} \mathrm{Mn}_{0.4} \mathrm{Co}_{0.2} \mathrm{O}_{2}$ 电池材料颗粒的化学成分和价态在三维空间的分布 ${ }^{[68]}$

Fig. $193 \mathrm{D}$ distributions of chemical components and valence states of $\mathrm{LiNi}_{0.4} \mathrm{Mn}_{0.4} \mathrm{Co}_{0.2} \mathrm{O}_{2}$ battery particles studied by X-ray spectro-microscopy ${ }^{[68]}$

(a) Panel illustrating the data structure; (b) Panel showing the prior knowledge of a list of anticipated principal chemical species in the sample, which is indispensable for the chemical mapping in this approach. Panel (c) illustrates the quantification procedures including the linear combination fitting, the color coding, and the tomographic reconstruction. Panel (d) shows the 3D mesoscale structural and chemical heterogeneity of a cluster of partially reduced $\mathrm{NiO}$ electrode particles

加速器技术的进步不断推动同步辐射光源的完善。 事实上，国内外不断有新的同步辐射光源的立项建 设或原有同步辐射装置的升级改造。例如, SSRF二 期工程即将建成新的 16 条线站，这将进一步提高从 软 X 射线到硬 X 射线的连续宽能谱研究能力; BSRF 正在建设具有世界领先水平的第四代同步辐射衍射 极限光源(HEPS), 可容纳 90 条以上的高性能光束 线站，建成后将成为当今世界上发射度最低、亮度 最高的同步辐射光源; NSRL 也在规划建设合肥先 进光源(HALF)。此外, 国内还有其他的同步辐射光 源也在规划中, 预计未来的 15 年是中国同步辐射光 源建设与发展的高光时期。

同步辐射实验技术已成为现代科学技术不可或 缺的研究手段。一方面, 同步辐射技术在科学研究 中的重要性日益增加, 越来越多的用户和结构表征 需要借助同步辐射技术; 另一方面, 高端用户越来 越不满足于已有的同步辐射技术, 不断刺激先进实 验技术的发展。例如: 物质结构的同步辐射表征从 静态过渡到原位动态演化; 单一尺度结构信息的获 取发展到多维度多级结构的同步表征; 常见表征手 段扩展到相干、瞬态、异常、精细等各种新的实验 技术。可以预计随着原位条件、实时动态、纳观分 辨、相干散射、技术联用等各种新实验技术不断完
善、提高和产生，比如，为同步得到材料的长程有序 和短程有序结构, 就需发展 XAFS/XRD 联用技术 ${ }^{[69]}$; 为了获取多种结构同步演化的信息，就需要发展 SAXS/WAXS/XAFS、SAXS/WAXS/Raman、SAXS/WAXS/R、 GISAXS/UV-Vis 等多种同步辐射联用技术或同步辐 射与非同步辐射的联用技术 ${ }^{[70]}$ 。

无机材料是同步辐射技术的主要应用领域之一， 同步辐射实验技术的应用势必极大地推动无机材料 的研究进展，提升我国无机材料研究的综合实力和 国际竞争力, 助力于解决无机材料领域中的一些关 键科学问题。

\section{参考文献:}

[1] 郑昌琼, 再均国. 新型无机材料. 北京: 科学出版社, 2003.

2] ZHANG L L, JIANG H L, CHEN M Q, et al. Nanotechnology and nanomaterial-application of inorganic nano-materials in traditional industry sectors. China Surfactant Detergent \& Cosmetics, 2004, 34(2): 123-126.

[3] KUMAR N, FOSSO-KANKEU E, RAY S S. Achieving controllable $\mathrm{MoS}_{2}$ nanostructures with increased interlayer spacing for efficient removal of $\mathrm{Pb}$ (II) from aquatic systems. ACS Applied Materials \& Interfaces, 2019, 11(21): 19141-19155.

[4] LIU Y P, WANG H, QIAN L X, et al. Bismuth-iron-based precursor: preparation, phase composition, and its thermal treatment in two way. RSC Advances, 2020, 10(35): 20713-20723.

[5] LIU Y P, QIAN L X, ZHAO X Y, et al. Synthesis and formation mechanism of self-assembled $3 \mathrm{D}$ flower-like $\mathrm{Bi} / \gamma-\mathrm{Fe}_{2} \mathrm{O}_{3}$ composite particles. CrystEngComm, 2019, 21(17): 2799-2808.

[6] WANG L, ZHOU $\mathrm{H}, \mathrm{HU}$ J, et al. A $\mathrm{Eu}^{3+}-\mathrm{Eu}^{2+}$ ion redox shuttle imparts operational durability to $\mathrm{Pb}$-I perovskite solar cells. Science, 2019, 363(6424): 265-270. 
[7] ZHAO X Y, LIU Y P, WANG J Y, et al. Modulating the hydrothermal synthesis of $\mathrm{CO}_{3} \mathrm{O}_{4}$ and $\mathrm{CoOOH}$ nanoparticles by $\mathrm{H}_{2} \mathrm{O}_{2}$ concentration. Inorganic Chemistry, 2019, 58(10): 7054-7061.

[8] LIU X J, HE J, ZHAO S Z, et al. Self-powered $\mathrm{H}_{2}$ production with bifunctional hydrazine as sole consumable. Nature Communications, 2018, 9: 4365 .

[9] ZOU Z Y, HABRAKEN W J E M, MATVEEVA G, et al. A hydrated crystalline calcium carbonate phase: calcium carbonate hemihydrate. Science, 2019, 363(6425): 396-400.

[10] HE W K, WANG D Y, WU H J, et al. High thermoelectric performance in low-cost $\mathrm{SnS}_{0.91} \mathrm{Se}_{0.09}$ crystals. Science, 2019, 365(6460): 1418-1424.

[11] WANG Y, DAR M I, ONO L K, et al. Thermodynamically stabilized $\beta$-CsPbI ${ }_{3}$-based perovskite solar cells with efficiencies> 18\%. Science, 2019, 365(6453): 591-595.

[12] SURYANARAYANA C. Structure and properties of nanocrystalline materials. Bulletin of Materials Science, 1994, 17(4): 307-346.

[13] KENDALL K. Materials Science and Technology, Volume 11: Structure and Properties of Ceramics. VCH, Weinheim, Germany: MV Swain, 1994: 841.

[14] WEN W, ZHANG L J, FU Y N, et al. Application of Shanghai light source in material science. Modern Physics, 2019, 31(5): 9-26.

[15] 冼鼎昌. 神奇的光: 同步辐射. 长沙: 湖南教育出版社, 1994.

16] ELDER F R, GUREWITSCH A M, LANGMUIR R V, et al. Radiation from electrons in a synchrotron. Physical Review, 1947, 71(11): 829-830.

[17] WINICK H, BIENENSTOCK A. Synchrotron radiation research. Annual Review of Nuclear and Particle Science, 1978, 28(1): 33-113.

[18] JACKSON JD. 经典电动力学(下册). 北京: 人民教育出版社, 1980

[19] 刘祖平. 同步辐射光源物理引论. 合肥: 中国科学技术大学出 版社, 2009.

[20] WINICK H, DONIACH S. Synchrotron radiation research. Journal of Vacuum Science and Technology, 1981, 19(1): 126.

[21] BROWN G S, MONCTON D E. Handbook on Synchrotron Radiation. North-Holland, 1991.

[22] 马礼敦, 杨福家. 同步辐射应用概论. 上海: 复旦大学出版社, 2001

[23] 姜晓明,修立松. 同步辐射及其应用. 北京: 北京科学技术出版 社, 1996.

[24] MAI Z H. Development history and current situation of synchrotron radiation-introduction to the new book "synchrotron radiation source and its application". Modern Physics, 2014, 26(2): 65-71.

[25] 北京同步辐射装置[EB/OL]. [2020-12-07]. http://www.ihep.cas. $\mathrm{cn} / \mathrm{dkxzz} / \mathrm{bsrf} /$

[26] 上海光源[EB/OL]. [2020-12-07]. http://ssrf.sinap.ac.cn/.

[27] 国家同步辐射实验室[EB/OL]. [2020-12-07]. http://www.nsrl.ustc. edu.cn/.

[28] MCCUSKER L B, VON DREELE R B, COX D E, et al. Rietveld refinement guidelines. Journal of Applied Crystallography, 1999, 32(1): $36-50$

[29] ZHAO H F, TAN D Y, TIAN Y, et al. Studies on Im-3-type $\mathrm{KSbO}_{3}$ using high pressure X-ray diffraction and Raman spectroscopy. High Pressure Research, 2018, 38(3): 232-242.

[30] YAN Z P, YIN K T, YU Z H, et al. Pressure-induced band-gap closure and metallization in two-dimensional transition metal halide $\mathrm{CdI}_{2}$. Applied Materials Today, 2020, 18: 100532

[31] FENG G Q, ZHANG W X, DONG L Y, et al. Negative area compressibility of a hydrogen-bonded two-dimensional material. Chemical Science, 2019, 10(5): 1309-1315.

[32] MAO H K, HEMLEY R J, WU Y, et al. High-pressure phase diagram and equation of state of solid helium from single-crystal X-ray diffraction to $23.3 \mathrm{GPa}$. Physical Review Letters, 1988, 60(25): 2649-2652

[33] FANG Y Y, ZHANG L, WU L W, et al. Pressure-induced emission (PIE) and phase transition of a two-dimensional halide double perovskite $(\mathrm{BA})_{4} \mathrm{AgBiBr}_{8} \quad\left(\mathrm{BA}=\mathrm{CH}_{3}\left(\mathrm{CH}_{2}\right)_{3} \mathrm{NH}^{3+}\right)$. Angewandte Chemie International Edition, 2019, 131(43): 15393-15397.

[34] ZUO Y X, LI B, JIANG N, et al. A high-capacity $\mathrm{O}_{2}$-type Li-rich cathode material with a single-layer $\mathrm{Li}_{2} \mathrm{MnO}_{3}$ superstructure. Advanced Materials, 2018, 30(16): 1707255.

[35] WANG J K, GAO R, ZHOU D, et al. Boosting the electrocatalytic activity of $\mathrm{Co}_{3} \mathrm{O}_{4}$ nanosheets for a $\mathrm{Li}-\mathrm{O}_{2}$ battery through modulating inner oxygen vacancy and exterior $\mathrm{Co}^{3+} / \mathrm{Co}^{2+}$ ratio. ACS Catalysis, 2017, 7(10): 6533-6541.

[36] WANG J K, GAO R, ZHENG L R, et al. $\mathrm{CoO} / \mathrm{CoP}$ heterostructured nanosheets with an O-P interpenetrated interface as a bifunctional electrocatalyst for $\mathrm{Na}_{2}$ battery. ACS Catalysis, 2018, 8(9): 8953-8960.

[37] DU R, CHEN, Z J, CAI Q, et al. Application of Mythen detector: in-situ XRD study on the thermal expansion behavior of metal indium. Science China-Physics Mechanics \& Astronomy, 2016, 59(7): 677011.

[38] DU R, CAI Q, CHEN Z J, et al. Mythen detector for X-ray diffraction at the Beijing synchrotron radiation facility. Instrumentation Science \& Technology, 2016, 44(1): 1-11.

[39] CHANG C, WU M H, HE D S, et al. 3D charge and 2D phonon transports leading to high out-of-plane ZT in n-type SnSe crystals. Science, 2018, 360(6390): 778-783.

[40] KRISHNAMURTI P. Studies in X-ray diffraction. Part I: the structure of amorphous scattering. Part II: colloidal solutions and liquid mixtures. Indian Journal of Physics, 1930, 5: 473-500.

[41] WARREN B E. X-ray diffraction study of carbon black. The Journal of Chemical Physics, 1934, 2(9): 551-555.

[42] ANDRÉ G, GÉRARD F, WALKER C B, et al. Small-angle scattering of X-rays. Physics Today, 1956, 9(8): 38

[43] YAN S, WU Z H, YU H Y, et al. Time-resolved small-angle X-ray scattering study on the growth behavior of silver nanoparticles. The Journal of Physical Chemistry C, 2014, 118(21): 11454-11463.

[44] YAN S, SUN D B, TAN Y Y, et al. Synthesis and formation mechanism of Ag-Ni alloy nanoparticles at room temperature. Journal of Physics and Chemistry of Solids, 2016, 98: 107-114.

[45] YAN S, SUN D B, GONG Y, et al. Temperature-driven directional coalescence of silver nanoparticles. Journal of Synchrotron Radiation, 2016, 23(3): 718-728.

[46] TAN Y Y, SUN D B, YU H Y, et al. In-situ time-resolved X-ray absorption fine structure and small angle $\mathrm{X}$-ray scattering revealed an unexpected phase structure transformation during the growth of nickel phosphide nanoparticles. The Journal of Physical Chemistry C, 2018, 122(28): 16397-16405.

[47] WANG J Y, ZHAO X Y, LIU Y P, et al. Small-angle X-ray scattering study on the orientation of suspended sodium titanate nanofiber induced by applied electric field. Radiation Detection Technology and Methods, 2019, 3(3): 1-7.

[48] LI H, LI C R, SUN W, et al. Single-stimulus-induced modulation of multiple optical properties. Advanced Materials, 2019, 31(23): 1900388.

[49] ZHANG H, DASBISWAS K, LUDWIG N B, et al. Stable colloids in molten inorganic salts. Nature, 2017, 542(7641): 328-331.

[50] YAO T, LIU S J, SUN Z H, et al. Probing nucleation pathways for morphological manipulation of platinum nanocrystals. Journal of the American Chemical Society, 2012, 134(22): 9410-9416.

[51] QIAO B T, WANG A Q, YANG X F, et al. Single-atom catalysis of CO oxidation using $\mathrm{Pt}_{1} / \mathrm{FeO}_{x}$. Nature Chemistry, 2011, 3(8): $634-641$.

[52] WANG X J, WANG H Y, LIU Y C, et al. A direct sulfation method for introducing the transition metal cation $\mathrm{Co}^{2+}$ into $\mathrm{ZrO}_{2}$ with little change in the Brønsted acid sites. Journal of Catalysis, 2011, 279(2): 301-309.

[53] XIN Y, LI H, ZHANG N N, et al. Molecular-level insight into selective catalytic reduction of $\mathrm{NO}_{x}$ with $\mathrm{NH}_{3}$ to $\mathrm{N}_{2}$ over a highly efficient bifunctional $\mathrm{Va}-\mathrm{MnO}_{x}$ catalyst at low temperature. ACS Catalysis, 2018, 8(6): 4937-4949.

[54] CAO L N, LIU W, LUO Q Q, et al. Atomically dispersed iron hydroxide anchored on $\mathrm{Pt}$ for preferential oxidation of $\mathrm{CO}$ in $\mathrm{H}_{2}$. Nature, 2019, 565(7741): 631-635.

[55] XIONG F Y, AN Q Y, XIA L X, et al. Revealing the atomistic origin of the disorder-enhanced Na-storage performance in $\mathrm{NaFePO}_{4}$ battery cathode. Nano Energy, 2019, 57: 608-615.

[56] LI B, JIANG N, HUANG W F, et al. Thermodynamic activation of charge transfer in anionic redox process for Li-ion batteries. Advanced Functional Materials, 2018, 28(4): 1704864

[57] HE W, WANG D, WU H, et al. High thermoelectric performance in low-cost $\mathrm{SnS}_{091} \mathrm{Se}_{0.09}$ crystals. Science, 2019, 365(6460): 1418-1424.

[58] HUMMEL R E. Electronic Properties of Materials. New York: Springer Science \& Business Media, 2011.

[59] LI J M, LEI T, WANG J O, et al. Anisotropic electronic structure and interfacial chemical reaction of stanene $/ \mathrm{Bi}_{2} \mathrm{Te}_{3}$. The Journal of Physical Chemistry C, 2020, 124(8): 4917-4924.

[60] WANG Z, FAN C C, SHEN Z X, et al. Defects controlled hole doping and multivalley transport in SnSe single crystals. Nature Communications, 2018, 9: 47.

[61] WANG F X, GUO J Z, WANG S X, et al. Yellow persistent luminescence and electronic structure of $\mathrm{Ca}-\alpha$-Sialon: $\mathrm{Eu}^{2+}$. Journal of Alloys and Compounds, 2020, 821: 153482.

[62] ZHOU X J, ZHONG J J, DONG J M, et al. Applications of NFPS/SSRF BL01B1 Synchrotron Infrared Beamline Station. Spectroscopy and Spectral Analysis, 2018, 38(10): 29-30.

[63] YUAN Q X, DENG B, GUAN Y, et al. Novel developments and applications of nanoscale synchrotron radiation microscopy. Physics, 2019, 48(4): 205-218

[64] CHEN J, WU C Y, TIAN J P, et al. Three-dimensional imaging of a complex concaved cuboctahedron copper sulfide crystal by X-ray nanotomography. Applied Physics Letters, 2008, 92(23): 233104.

[65] CAO C, TONEY M F, SHAM T K, et al. Emerging X-ray imaging technologies for energy materials. Materials Today, 2020, 34: 132-147.

[66] QU J, CAO C Y, HONG Y L, et al. New hierarchical zinc silicate nanostructures and their application in lead ion adsorption. Journal of Materials Chemistry, 2012, 22(8): 3562-3567.

[67] LIN F, NORDLUND D, LI Y Y, et al. Metal segregation in hierarchically structured cathode materials for high-energy lithium batteries. Nature Energy, 2016, 1: 15004.

[68] WEI C X, XIA S H, HUANG H, et al. Mesoscale battery science: the behavior of electrode particles caught on a multispectral X-ray camera. Accounts of Chemical Research, 2018, 51(10): 2484-2492.

[69] LIU Z J, LUO Z L. Simultaneous quick measurements of combined synchrotron EDXAFS and EDXRD. Nuclear Techniques, 2019, 42(12): 8-12.

[70] WU Z H, HUANG Y Y, ZHANG J L, et al. Real-time tracking research on the formation of new materials in complex fluids. China Basic Science, 2018, 20: 17-22. 